ARTICLE

\title{
Deacetylation as a receptor-regulated direct activation switch for pannexin channels
}

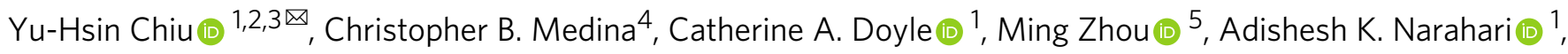
Joanna K. Sandilos', Elizabeth C. Gonye (1) 1, Hong-Yu Gao², Shih Yi Guo ${ }^{3}$, Mahmut Parlak (1) 4, Ulrike M. Lorenz ${ }^{4}$, Thomas P. Conrads (1) ${ }^{5}$, Bimal N. Desai ${ }^{1}$, Kodi S. Ravichandran ${ }^{4} \&$ Douglas A. Bayliss (iD) ${ }^{1 \times}$

Activation of Pannexin 1 (PANX1) ion channels causes release of intercellular signaling molecules in a variety of (patho)physiological contexts. PANX1 can be activated by G proteincoupled receptors (GPCRs), including $\alpha 1$-adrenergic receptors ( $\alpha 1$-ARs), but how receptor engagement leads to channel opening remains unclear. Here, we show that GPCR-mediated PANX1 activation can occur via channel deacetylation. We find that $\alpha 1-A R-m e d i a t e d$ activation of PANX1 channels requires G $\alpha$ q but is independent of phospholipase $C$ or intracellular calcium. Instead, $\alpha 1-A R-m e d i a t e d ~ P A N X 1$ activation involves RhoA, mammalian diaphanous (mDia)-related formin, and a cytosolic lysine deacetylase activated by mDia - histone deacetylase 6. HDAC6 associates with PANX1 and activates PANX1 channels, even in excised membrane patches, suggesting direct deacetylation of PANX1. Substitution of basallyacetylated intracellular lysine residues identified on PANX1 by mass spectrometry either prevents HDAC6-mediated activation (K140/409Q) or renders the channels constitutively active (K140R). These data define a non-canonical RhoA-mDia-HDAC6 signaling pathway for G $\alpha q P C R$ activation of PANX1 channels and uncover lysine acetylation-deacetylation as an ion channel silencing-activation mechanism.

\footnotetext{
${ }^{1}$ Department of Pharmacology, University of Virginia, Charlottesville, VA, USA. ${ }^{2}$ Institute of Biotechnology, National Tsing Hua University, Hsinchu, Taiwan. ${ }^{3}$ Department of Medical Science, National Tsing Hua University, Hsinchu, Taiwan. ${ }^{4}$ Department of Microbiology, Immunology \& Cancer Biology, University of Virginia, Charlottesville, VA, USA. ${ }^{5}$ Inova Center for Personalized Health, Inova Schar Cancer Institute, Fairfax, VA, USA. ${ }^{凶}$ email: yhchiu@life.nthu.edu.tw; dab3y@virginia.edu
} 
annexin 1 (PANX1) forms oligomeric ion channels that are renowned for releasing nucleotides to mediate purinergic signaling in multiple (patho)physiological contexts ${ }^{1-3}$. Depending on the context, different channel activation mechanisms have been defined. During apoptosis, stepwise caspasemediated cleavage of PANX1 causes a graded, but irreversible, channel activation to support release of ATP and other metabolites that attract phagocytes to facilitate immunologically silent clearance of dying cells and establish a local anti-inflammatory environment ${ }^{4-7}$. There is also evidence for direct, reversible PANX1 activation by physicochemical factors such as membrane stretch (e.g., in cancer metastasis) ${ }^{8,9}$, high extracellular potassium (e.g., in seizures) ${ }^{10-12}$, or elevated intracellular calcium (e.g., in aberrant cardiomyocyte depolarization) ${ }^{13}$. In addition, indirect activation of PANX1 by $G$ protein-coupled receptors (GPCRs) has been implicated in numerous settings (e.g., injuryinduced neuropathic pain, airway irritant responses, blood pressure regulation, etc. ${ }^{14-17}$ ). However, the downstream signaling pathways and channel regulatory mechanisms that account for GPCR-mediated PANX1 activation have not been determined.

The GPCRs identified with PANX1 activation are typically associated with Gaq-containing heterotrimeric $\mathrm{G}$ proteins, and in some cases a role for intracellular $\mathrm{Ca}^{2+}$ has been suggested. For example, Panxl activation in Xenopus oocytes by co-expressed $\mathrm{P} 2 \mathrm{Y}$ receptors could be mimicked with a calcium ionophore or direct exposure of Panx1-containing membrane patches to elevated $\mathrm{Ca}^{2+18}$. However, a $\mathrm{Ca}^{2+}$ contribution to receptormediated Panx1 activation was not directly tested, and a mechanism by which $\mathrm{Ca}^{2+}$ might directly activate the channels was not defined. $\mathrm{A} \mathrm{Ca}^{2+}$-dependent mechanism was also implicated in PAR1-mediated activation of Panx1, for which concomitant actions of Rho-associated protein kinase (ROCK) were also required ${ }^{14}$; a specific mechanism by which ROCK contributed to Panx1 channel activation was not determined. It has also been suggested that phosphorylation of Tyr-198 of Panx1 might play a role in channel modulation by $\alpha 1-\mathrm{AR}^{15}$, but recent observations indicate that Y198 phosphorylation of Panx1 is unaffected by al-AR stimulation ${ }^{19}$. Thus, the receptor-mediated Panxl activation mechanisms remain elusive.

In this work, using molecular and pharmacological tools, we show that Panxl activation by the a1D-AR engages Gaq and an unconventional RhoA-mDia-HDAC6 signaling pathway to mediate direct activation of Panx1 channels by lysine deacetylation. The findings raise the possibility that acetylation-deacetylation may be a more general switch mechanism for ion channel activation. In addition, we demonstrate this al-AR and HDAC6mediated Panxl activation in $\mathrm{T}$ lymphocytes, suggesting a potential signaling modality for neuro-immune modulation by GPCRs $^{20}$.

\section{Results}

PANX1 channels are activated by Gaq-coupled receptors. We established a cellular reconstitution system in which co-expression of various Gaq-coupled receptors with either mouse or human PANX1 channels in HEK293T cells recapitulates receptormediated channel activation (Supplementary Fig. 1) ${ }^{5,15}$. We find that HEK293T cells have no detectable endogenous PANX1 currents (i.e., a null system), assessed using the channel blocker carbenoxolone $(\mathrm{CBX})^{5,15}$; when transfected in those cells, mPanx1 generates basal whole-cell currents whereas hPANX1 is basally silent (Supplementary Fig. 1a-c). mPanx1 and hPANX1 are both activated by co-expressed $\alpha 1 \mathrm{D}$-adrenergic receptor ( $\alpha 1 \mathrm{D}-\mathrm{AR}$; Supplementary Fig. $1 \mathrm{a}-\mathrm{c}$ ), and mPanx1 is activated by multiple additional GaqPCRs (e.g., H1 histamine receptor, metabotropic glutamate receptor mGluR1; Supplementary Fig. 1e, f). Note that Panx 1 channels are active over a wide voltage range and generate a weakly outwardly rectifying, open channel current (i.e., evident in $I-\mathrm{V}$ curves as large outward current at positive membrane potentials, with smaller, but clearly evident inward current at negative potentials $)^{5}$.

In a1D-AR-expressing cells, mPanxl channel activation by the a1-selective agonist phenylephrine (PE) was observed in cellattached patches (Fig. 1a, b) ${ }^{5}$. In this recording configuration, the agonist-stimulated receptor is physically separated from the Panx 1 channels that are recorded within the membrane patch, thus implicating a diffusible signaling pathway. The a1D-evoked currents appear to be due to effects on channels already located on the cell membrane since prolonged agonist treatment did not appreciably alter cell-surface levels of the channel (Supplementary Fig. 1d). Importantly, a1D-mediated activation was fully intact in channels bearing a mutation in the PANX1 caspase cleavage site (Supplementary Fig. 1b, c), and it was reversible upon wash of PE (Supplementary Fig. 1c), indicating that it is independent of the irreversible cleavage-based channel activation mechanism we previously described ${ }^{4-6}$.

In a1D-AR-expressing cells, whole-cell mPanxl currents were activated by $\mathrm{PE}$ when recorded using pipettes containing GTP but not those containing GDP $\beta$, a GDP analog that interferes with receptor-mediated Ga-GDP:GTP exchange (Fig. 2a-c). In addition, and as expected for this Gaq-coupled receptor, we found that PE-mediated stimulation of a1D-AR did not activate Panx1 channels expressed in fibroblasts derived from Gaq/11 doubleknockout mice (Fq11 cells), but receptor-mediated channel activation was rescued when Gaq was re-expressed in those cells (Fig. 2d, e, g). Notably, receptor-mediated Panx1 activation in Fq11 cells was not rescued by co-expression of a mutated Gaq (A253K) construct (Fig. 2f, g) that is unable to couple to p63RhoGEF ${ }^{21}$ but retains the ability to activate PLC (i.e., increase intracellular calcium) (Fig. 2h, i). This indicates that PLC activation is not sufficient for Panxl activation; the lack of rescue

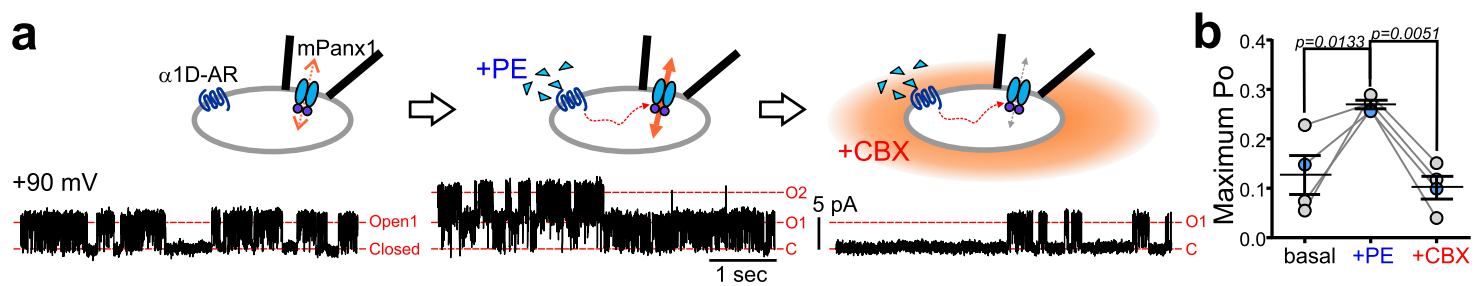

Fig. 1 Panx1 channel activation by $\alpha 1 D-A R s$ requires a diffusible messenger. a Exemplar cell-attached patch recording shows phenylephrine (PE, $20 \mu \mathrm{M}$ )induced activation of carbenoxolone (CBX, $50 \mu \mathrm{M}$ )-sensitive channels in HEK293T cells expressing $\alpha 1 D$-adrenergic receptors ( $\alpha 1 D$-ARs) and mPanx1. The schematic illustrates that, in this configuration, the liganded $\alpha 1 \mathrm{D}$ receptor is physically separated from the recorded channel in the patch pipette, activation of which must involve a diffusible messenger. $\mathbf{b}$ Effects of PE and CBX on channel activity (maximum $P_{O}$ ) in those cell-attached patches ( $n=4$ membrane patches examined over 4 independent experiments). Data from the exemplar patch recording (in a) is represented by cyan dots. One-way ANOVA ( $F_{2,9}=$ $11.35, p=0.0035$ ) with Bonferroni's multiple comparisons test ( $p$ values from comparisons are shown). 

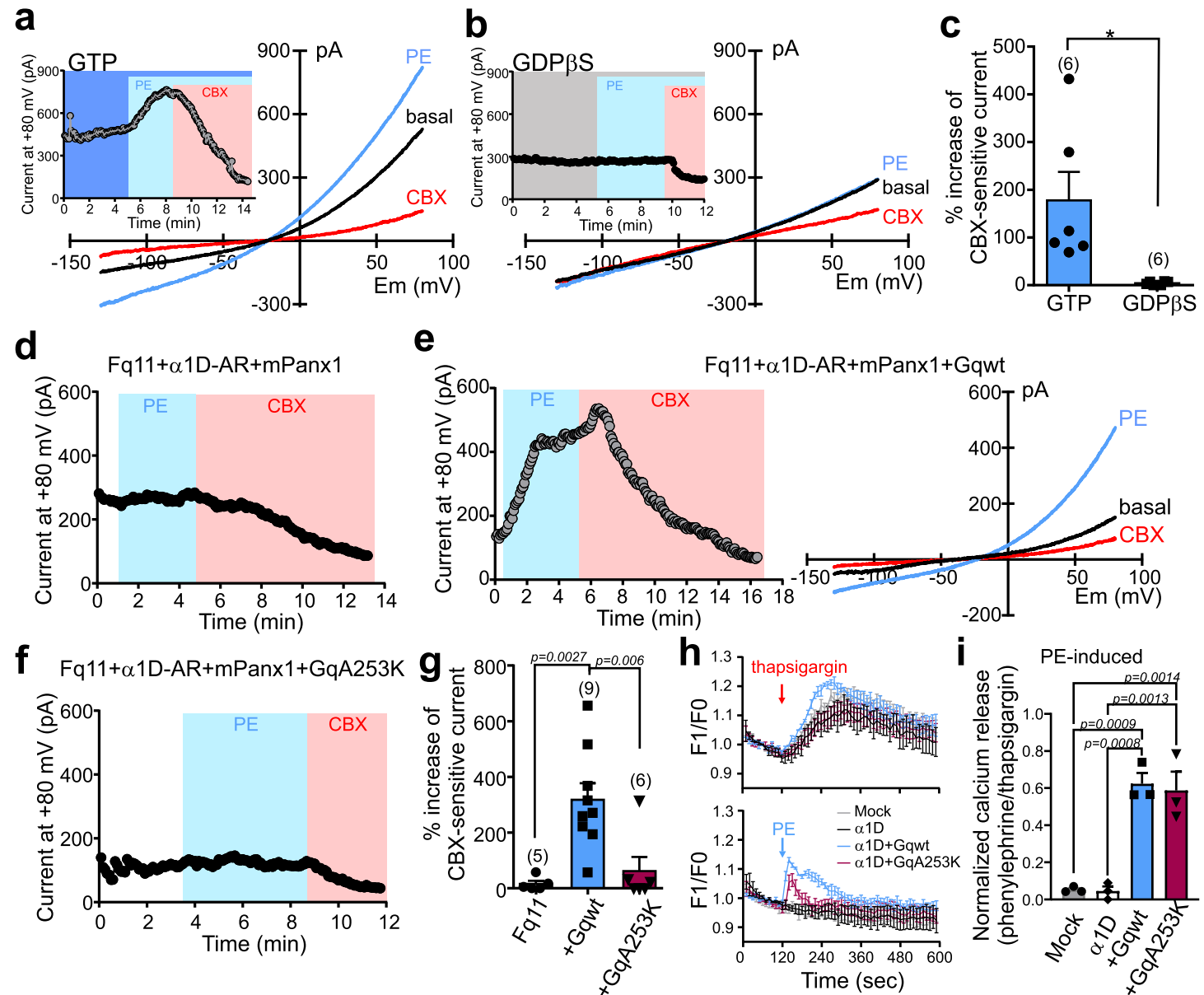

$-300$
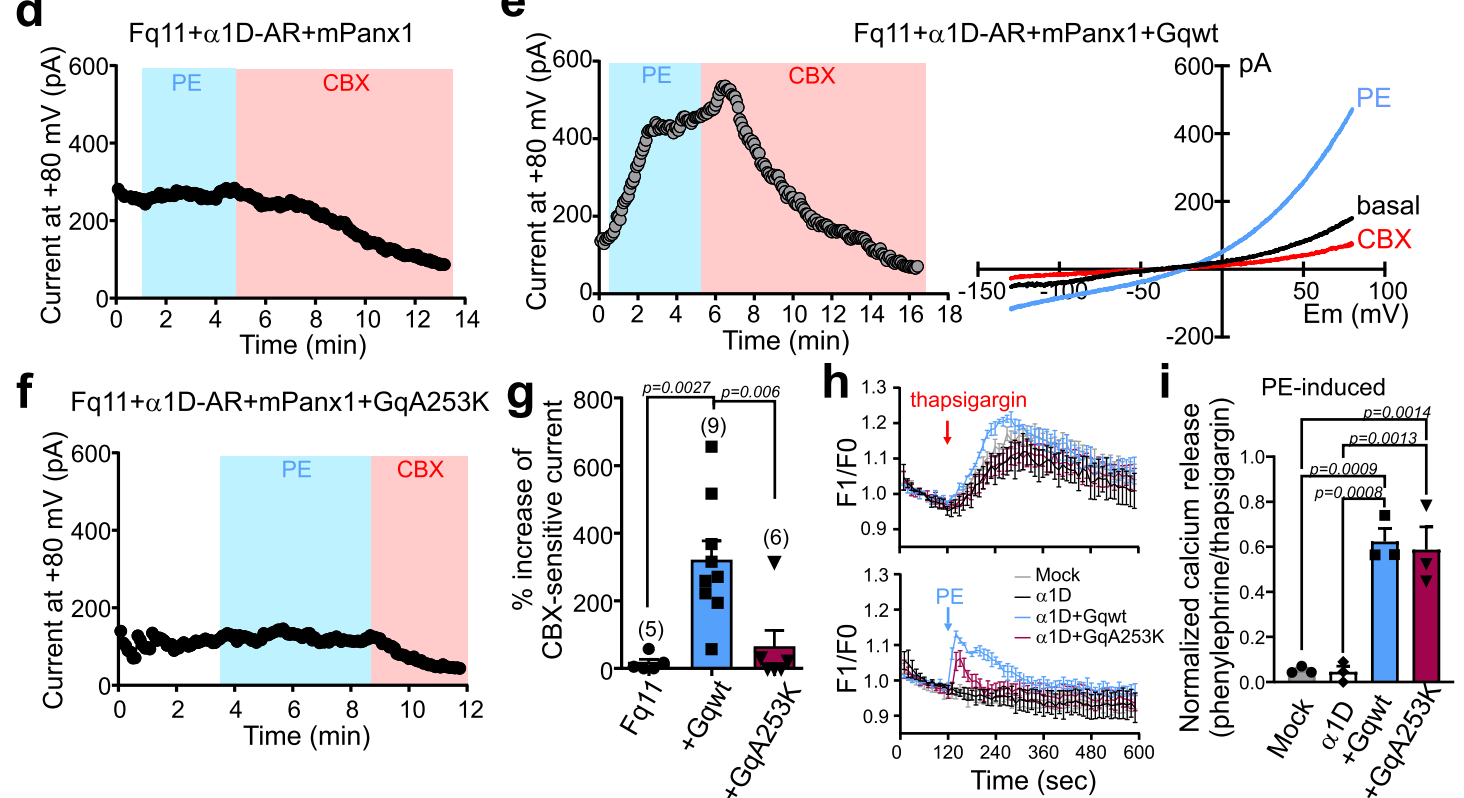

Fig. 2 Goq proteins are required for Panx1 channel activation by $\alpha 1 D-A R s . ~ a-c$ Effect of PE on whole-cell mPanx1 currents in $\alpha 1 D-A R / m P a n \times 1-t r a n s f e c t e d$ cells recorded with either GTP (a) or GDPBS (b) in the pipette. I-V relationships depicted are from ramp voltage commands and time series from current amplitude at $80 \mathrm{mV}$ (Insets). Grouped data (c) show that GDPBS diminished PE-induced current. PE-induced whole-cell current was normalized to basal CBX-sensitive current. ${ }^{\star} p=0.0160$ using two-tailed $t$-test. $n=6$ cells per group examined over 3 independent experiments. $\mathbf{d}$-f Effect of PE on whole-cell $\mathrm{mPanx} 1$ currents in fibroblasts from $\mathrm{G} \alpha \mathrm{q} / 11$ double-knockout mice (Fq11) transfected with $\alpha 1 \mathrm{D}-\mathrm{AR}$ and $\mathrm{mPanx1}$ alone (d), or when co-transfected with wildtype $G \alpha q(\mathbf{e})$ or $G \alpha q(A 253 K)$ (f). $\mathbf{g}$ Summary data reveal that only wild-type G $\alpha q$ could rescue PE activation of mPanx1 in Fq11 cells. $n=5$, 9 , or 6 cells examined over 3 independent experiments. One-way ANOVA $\left(F_{2,5}=11.63, p=0.0009\right)$ with Bonferroni's multiple comparisons test ( $p$ values from

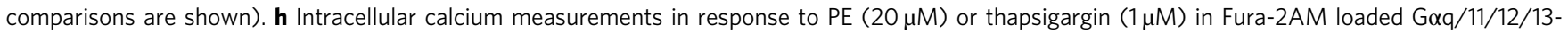
deleted HEK293 cells transfected with $\alpha 1 D-A R$, with or without different G $\alpha$ q proteins ( $n=3$ biologically independent experiments). i Averaged peak signal for receptor-mediated calcium transients normalized to thapsigargin $\left(n=3\right.$ biologically independent experiments). One-way ANOVA $\left(F_{3,8}=28.57, p=\right.$ $0.0001)$ with Bonferroni's multiple comparisons test ( $p$ values from comparisons are shown). Summary data are presented throughout as mean \pm s.e.m. $P E$ phenylephrine.

with $\mathrm{Gaq}(\mathrm{A} 253 \mathrm{~K})$ suggests instead that there may be a role for Gaq-mediated Rho activation.

We also found that receptor-mediated Panx1 activation was largely unaffected when cells were recorded with pipettes containing high concentrations of BAPTA to rapidly chelate intracellular calcium ( $\mathrm{pCa} \sim 8$ ), again consistent with the idea that a $\mathrm{Ca}^{2+}$-dependent signaling pathway is dispensable for channel activation (Supplementary Fig. 2a, b). This is also in line with previous work reporting that PE-mediated ATP release from cells co-expressing $\alpha 1 \mathrm{D}-\mathrm{AR}$ and Panx1 was unaffected by intracellular calcium chelation ${ }^{19}$. Collectively, these results support a Gaqmediated, diffusible signaling pathway interposed between a1D$\mathrm{AR}$ and activation of Panx1 channels; this pathway is independent of PLC-Ca ${ }^{2+}$ and may involve activation of RhoA.

RhoA is necessary and sufficient for activation of PANX1 channels. To examine whether RhoA is required for a1Dmediated Panx 1 activation, we tested effects of two Rho inhibitors with different mechanisms of action: C3 exoenzyme, which ADP- ribosylates and inactivates Rho family proteins ${ }^{22}$; and RhoA (T19N), which is a dominant-negative form of $\mathrm{RhoA}^{23,24}$. In HEK293T cells expressing a1D-AR and mPanx1, PE-activated whole-cell currents were strongly reduced when either C3 exoenzyme or RhoA(T19N) were co-transfected (Fig. 3a-c). To establish whether RhoA can act downstream of the a1D-AR receptor to activate Panxl currents, we co-expressed a constitutively active RhoA(G14V) construct in hPANX1-expressing cells $^{23,25}$. Strikingly, basal PANX1 currents were already elevated upon whole-cell access in cells co-expressing RhoA(G14V), and a1D-AR stimulation by PE did not further activate the current (Fig. 3d, g). This effect was not observed in control experiments using a RhoA $(\mathrm{G} 14 \mathrm{~V})$ construct that was further mutated to disrupt interactions with all known RhoA effectors (Supplementary Fig. 2f $)^{23,25}$; that is, in RhoA(G14V/T37Y)-expressing cells, PE evoked the characteristic increase in PANX1 current from low to undetectable baseline levels (Fig. $3 \mathrm{e}, \mathrm{g}$ ). These data indicate that RhoA activation is necessary and sufficient for a1D-mediated PANX1 activation. 
a

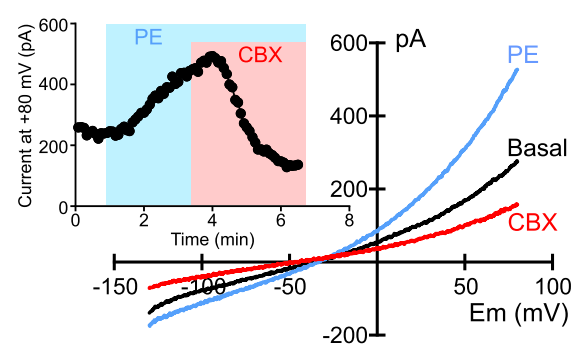

d a1D-AR+hPANX1(TEV)+RhoA(G14V)

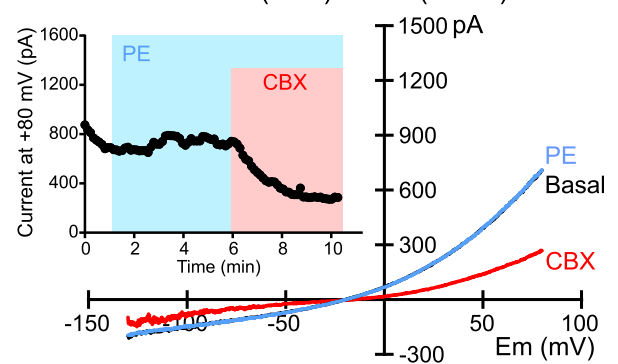

$\mathbf{f}_{\alpha 1 \mathrm{D}-\mathrm{AR}+\mathrm{hPANX} 1(\mathrm{TEV})+\mathrm{RhoA}(\mathrm{G} 14 \mathrm{~V} / \mathrm{F} 39 \mathrm{~A})}$

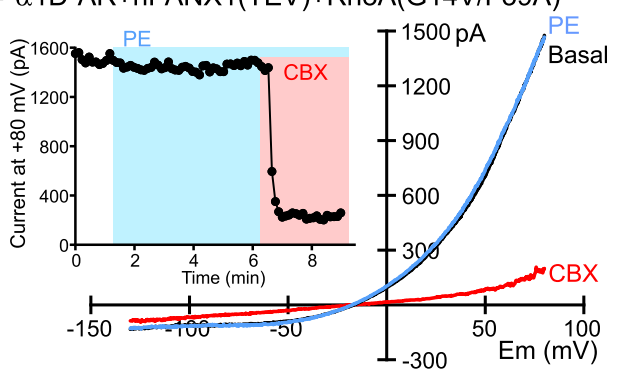

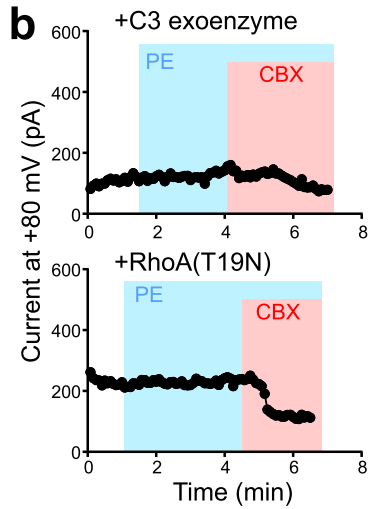

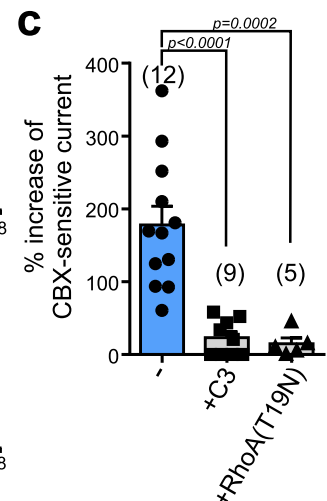

e $\alpha 1 D-A R+h P A N X 1(T E V)+R h o A(G 14 V / T 37 Y)$

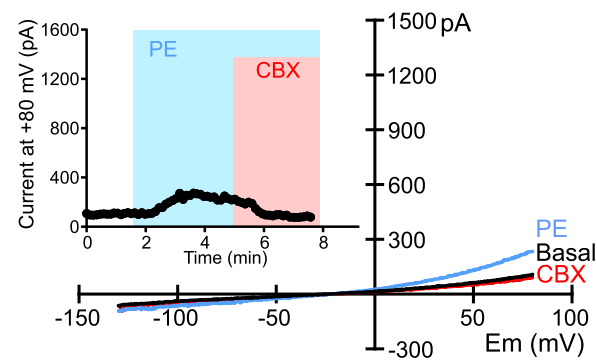

$\mathbf{g}$

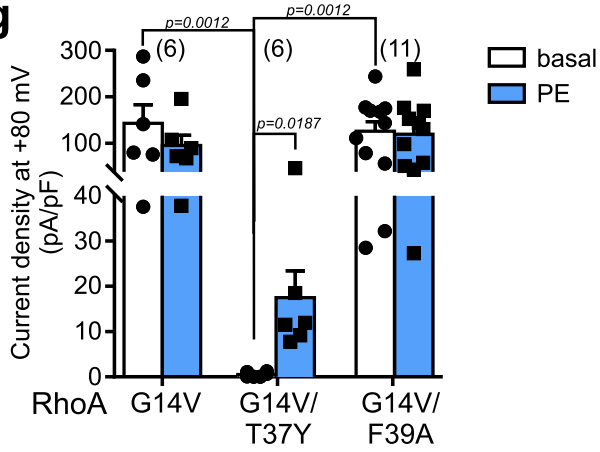

Fig. 3 RhoA is necessary and sufficient for receptor-mediated PANX1 activation. a, b Effect of PE on whole-cell currents in HEK293T cells transfected with $\alpha 1 \mathrm{D}-\mathrm{AR}$ and $\mathrm{mPanx1}$ (TEV) alone (a) or when co-transfected with either C3 exoenzyme or dominant-negative RhoA(T19N) (b). c Grouped data (mean \pm s.e.m) show that overexpression of $\mathrm{C} 3$ exoenzyme or RhoA(T19N) reduced PE-evoked Panx1 current. $n=12,9$, or 5 cells per group examined over 4 independent experiments. One-way ANOVA $\left(F_{2,23}=19.94, p<0.0001\right)$ with Bonferroni's multiple comparisons test. d-f Whole-cell currents in $\alpha 1 D$-AR/ hPANX1(TEV)-transfected cells co-expressing the constitutively-activated RhoA constructs: RhoA(G14V) (d), RhoA(G14V/T37Y), a control that cannot couple to downstream effectors (e), or RhoA(G14V/F39A) that only couples to mDia (f). $\mathbf{g}$ Summary data (mean \pm s.e.m) reveal that the mDia-activating RhoA(G14V/F39A) construct can recapitulate increased basal hPANX1 currents and occluded PE effects observed with RhoA(G14V). $n=6,6$, or 11 cells per group examined over 5 independent experiments. Two-way ANOVA $\left(F_{2,20}=7.776, p=0.0032\right)$ with Bonferroni's multiple comparisons test. PE phenylephrine.

Rho family GTPases have multiple downstream effectors, such as Rho-associated kinases (ROCK1, ROCK2), Src family kinases (SFKs), and mammalian diaphanous-related formins (mDia1 3) ${ }^{23,26-28}$. Activation of Panx1 channels by a1D-AR stimulation was unaffected by two chemically distinct ROCK inhibitors, Y27632 or H1152 (Supplementary Fig. 2c-e). This indicates that receptor-mediated channel activation can bypass ROCK, and implies involvement of a mechanism distinct from the Rho-ROCK-PANX1 pathway activated by hypotonic solution in airway epithelial cells ${ }^{24}$. Likewise, whereas SFKs are proposed to activate Panxl channels by phosphorylation at Tyr-198 or Tyr$308^{15,29}$, phenylalanine substitutions at those channel sites (Y198F/Y308F) did not disrupt Panx1 activation by a1D-AR stimulation (Supplementary Fig. 3). On the other hand, coexpression of RhoA(G14V/F39A), a variant of constitutively active RhoA that couples only to mDia (mDial 3) (Supplementary Fig. $2 \mathrm{f})^{23,25}$, was able to recapitulate effects of RhoA(G14V) in mimicking and occluding receptor-mediated PANX1 activation (Fig. 3f, g). Thus, we found large PANX1 currents under unstimulated conditions in cells transfected with RhoA(G14V/ F39A), and those currents were not further increased by PE stimulation. These data suggest that RhoA stimulation of PANX1 is independent of ROCK or SFKs, and may instead involve mDia.

mDia and HDAC6 mediate activation of PANX1 channels. Under unstimulated conditions, mDia proteins are prevented from interacting with target proteins by an intramolecular interaction involving the so-called diaphanous autoregulatory domain (DAD) ${ }^{30-32}$; this autoinhibition is released by Rho-GTP binding to a GTPase binding domain (GBD) of $\mathrm{mDia}$, and can also be undone experimentally by exogenous overexpression of a competing DAD mimetic peptide ${ }^{32}$. In cells expressing DAD, prominent basal PANX1 currents were observed under unstimulated conditions, and those currents were not further elevated 

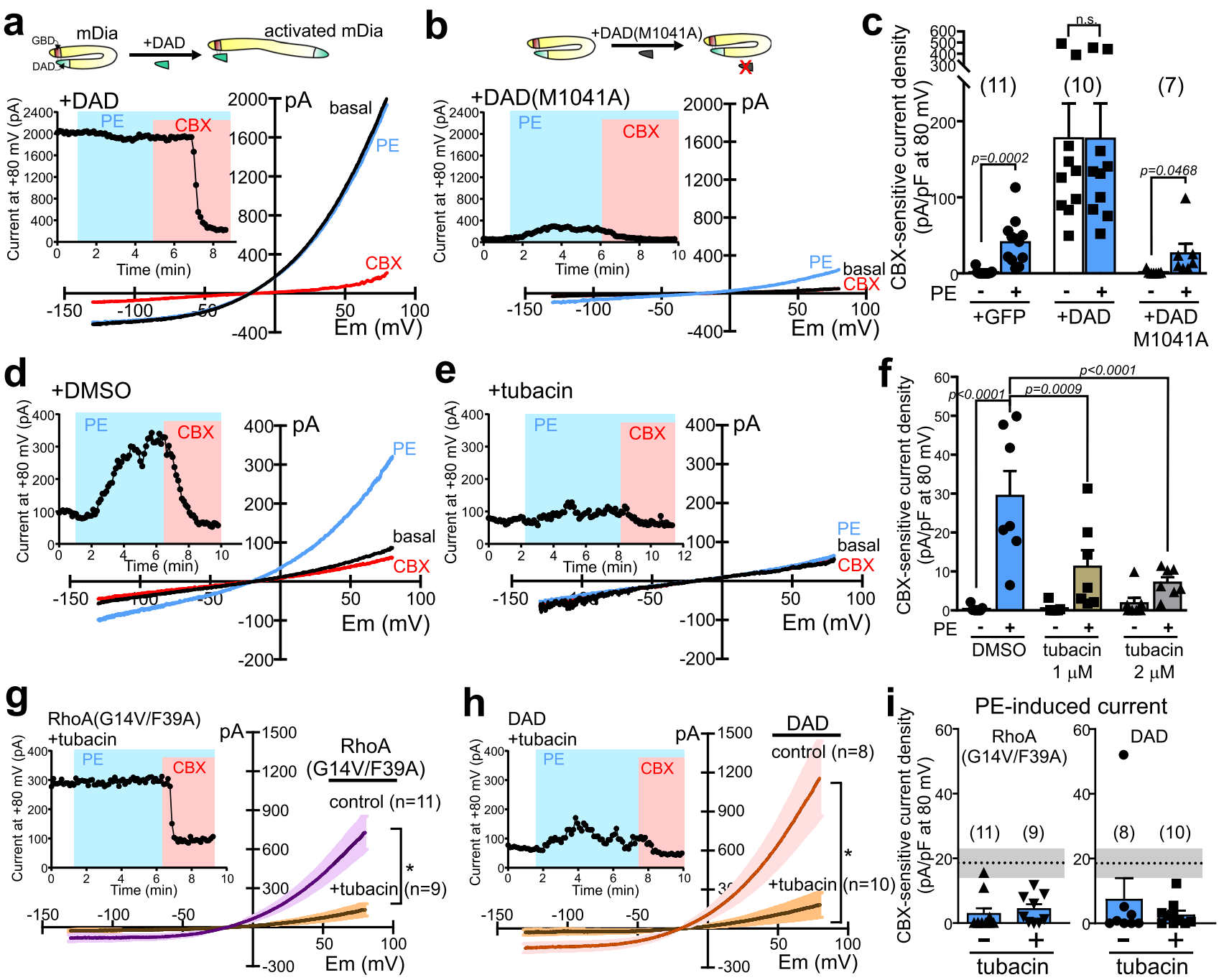

Fig. 4 mDia and HDAC6 mediate $\alpha 1 D-A R$ activation of PANX1. a, b Basal and PE-stimulated whole-cell currents in $\alpha 1 D-A R / h P A N X 1(T E V)-t r a n s f e c t e d$ HEK293T cells co-expressing a wild-type diaphanous autoregulatory domain (DAD) construct (a) or a mutated DAD(M1041A) version that cannot bind mDia (b). c Summary data (mean \pm s.e.m) reveal that DAD, but not DAD(M1041A), increased basal PANX1 current and occluded PE effects. $n=11,10$, or 7 cells examined over 5 independent experiments. Two-way ANOVA $\left(F_{2,25}=5.673, p=0.0093\right)$ with Holm-Sidak's multiple comparisons test ( $p$ values from comparisons are shown). n.s. not significant. $\mathbf{d}$, e Basal and PE-stimulated whole-cell currents in $\alpha 1 D$-AR/hPANX1-transfected cells recorded with vehicle (DMSO, $14.1 \mu \mathrm{M})$ or tubacin $(2 \mu \mathrm{M})$ in the pipette. $\mathbf{f}$ Concentration-dependent inhibition of PE-induced hPANX1 current by tubacin. $n=7$ cells per group examined over 5 independent experiments. Data are presented as mean \pm s.e.m. Two-way ANOVA $\left(F_{2,18}=7.636, p=0.0040\right)$ with Bonferroni's multiple comparisons test. $\mathbf{g}, \mathbf{h}$ Effects of tubacin $(2 \mu \mathrm{M})$ treatment on basal whole-cell currents (mean \pm s.e.m in $\alpha 1 D-A R / h P A N X 1(T E V)$-transfected cells co-expressing the mDia-activating constructs, RhoA(G14V/F39A) (g) or DAD (h). Tubacin was added in culture media after transfection, and to the intracellular solution for whole-cell recording. Insets: representative time series of whole-cell current at $80 \mathrm{mV}$ from $\alpha 1 \mathrm{D}-\mathrm{AR} / \mathrm{hPANX1(TEV)-transfected}$ cells, with RhoA(G14V/F39A) or DAD, treated with tubacin. i Summary data (mean \pm s.e.m) reveal that PE-induced CBX-sensitive currents from $\alpha 1 D$-AR/ hPANX1(TEV)-expressing cells were diminished by co-expression of RhoA(G14V/F39A) or DAD, regardless of tubacin treatment. $n=11,9,8$, or 10 cells examined over 6 independent experiments. Dotted line and shaded area indicate mean and SEM of PE-evoked, CBX-sensitive current density from cells expressing $\alpha 1 \mathrm{D}-\mathrm{AR}$ and hPANX1. PE phenylephrine, CBX carbenoxolone.

by a1D-AR receptor stimulation (Fig. $4 \mathrm{a}, \mathrm{c}$ ). These effects of DAD were remarkably similar to those obtained with constitutively active RhoA(G14V), including the F39A variant that interacts only with $\mathrm{mDia}$; they were not seen in control cells expressing a mutated $\mathrm{DAD}(\mathrm{M} 1041 \mathrm{~A})$ construct that cannot bind mDia (Fig. $4 b, c)^{32,33}$. These data indicate that activated forms of RhoA, as well as DAD constructs that reproduce RhoA-mediated relief of mDia autoinhibition, can mimic and occlude receptormediated PANX1 activation.

HDAC6 is a cytosolic, class IIb histone deacetylase that mediates Rho- and mDia-induced microtubule deacetylation in osteoclast cells ${ }^{34-36}$, suggesting that it could function as a downstream effector in a1D-AR-mediated Panxl activation.
Consistent with this, PE-induced Panx1 current was inhibited by trichostatin A, a broad-spectrum class I and class II HDAC inhibitor (Supplementary Fig. 4a, b), and by tubacin, a more selective HDAC6 inhibitor (Fig. 4d-f) 37,38 . Moreover, tubacin treatment blocked the increase in basal current that was observed in cells expressing the mDia activators, RhoA(G14V/F39A) or DAD (Fig. 4g, h). Notably, despite the much lower initial current after tubacin, PE was still unable to activate Panxl current (Fig. 4g-i). This suggests that the blunted a1D action in RhoA (G14V/F39A)- or DAD-expressing cells is not due to a "ceiling" effect but rather more likely represents convergence on a shared mechanism of action (i.e., occlusion). Together, these data suggest 

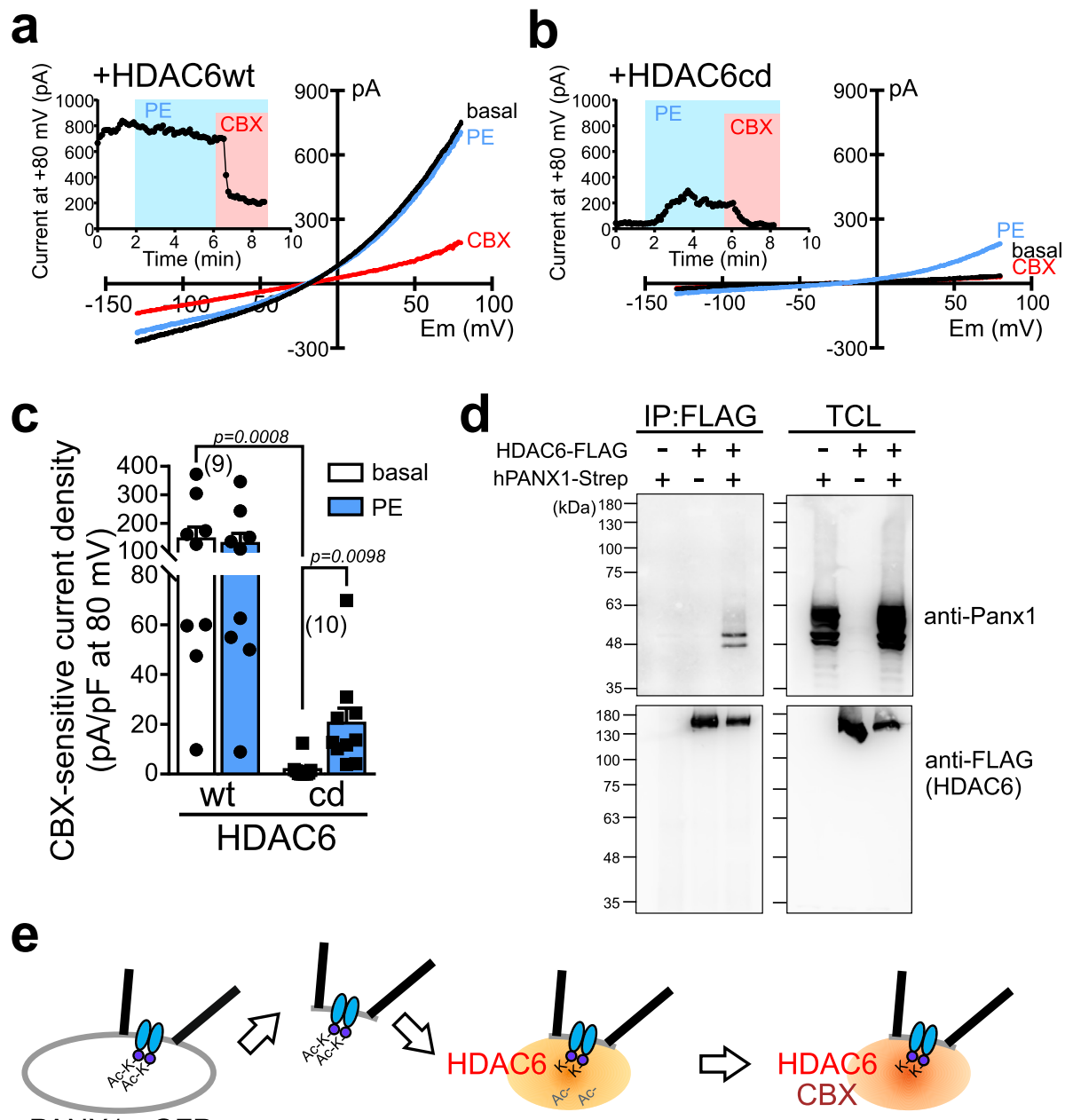

PANX1-eGFP
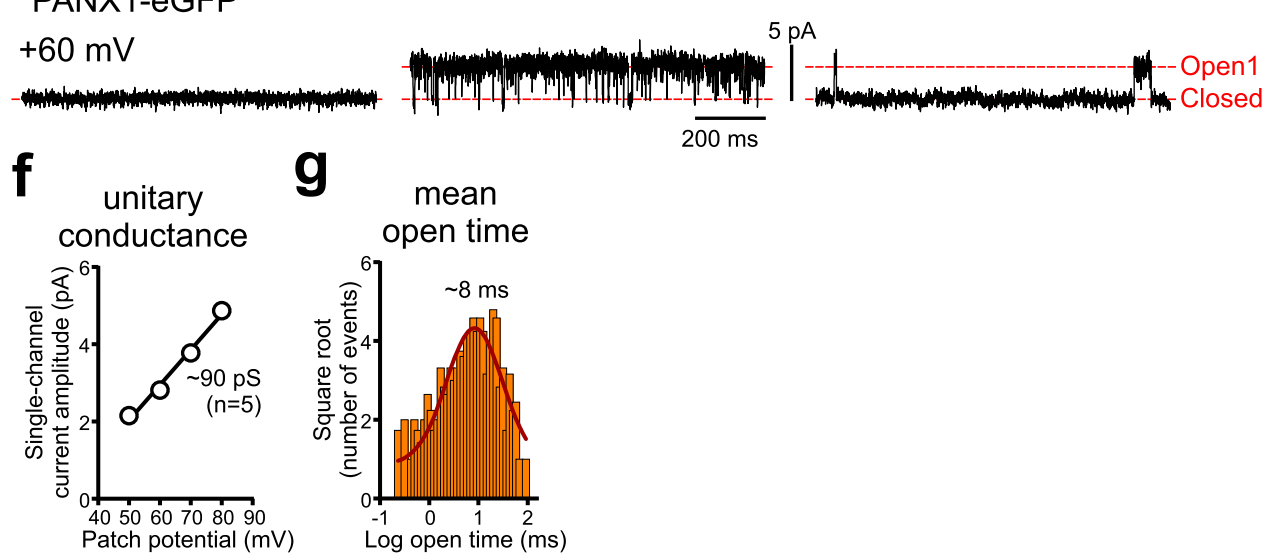

Fig. 5 HDAC6 is sufficient to activate PANX1, even in cell-free conditions. a, b Basal and PE-stimulated whole-cell currents in $\alpha 1 D-A R / h P A N X 1(T E V)$ transfected HEK293T cells co-expressing wild-type (a) or catalytically inactive (b) forms of HDAC6. c Summary data (mean \pm s.e.m) showing that the active form of HDAC6 increased basal PANX1 current and occluded PE effects. $n=9$ or 10 cells examined over 3 independent experiments. Two-way ANOVA $\left(F_{1,17}=18.07, p=0.0005\right)$ with Bonferroni's multiple comparisons test ( $p$ values from comparisons are shown). d Co-immunoprecipitation showing interaction between HDAC6 and hPANX1 (from $n=3$ ). FLAG-tagged HDAC6 and strep-tag-conjugated PANX1 were heterologously expressed in HEK293T cells. PANX1 proteins were co-immunoprecipitated with FLAG-tagged HDAC6. TCL total cell lysate. e Effect of HDAC6 and CBX on GFP-tagged hPANX1 channel recorded in inside-out patch configuration. $\mathbf{f}, \mathbf{g}$ Averaged unitary I-V (f) and a representative open time distribution (g) for HDAC6activated PANX1 channels in inside-out patches $(\gamma=89.5 \pm 1.8 \mathrm{pS}$, mean open time $=8.3 \pm 1.3 \mathrm{~ms}, n=5$ independent membrane patches; mean \pm s.e.m). $\mathrm{PE}$ phenylephrine, $\mathrm{CBX}$ carbenoxolone.

that HDAC6 may serve as an intermediary downstream of RhoA and $\mathrm{mDia}$ for a1D-AR-mediated PANX1 activation.

HDAC6 directly activates PANX1 channels by deacetylation. We next tested whether the cytosolic deacetylase HDAC6 can activate PANX1. Heterologous expression of HDAC6, but not a catalytically inactive HDAC6, yielded large basal whole-cell PANX1 currents and occluded effects of a1D-AR activation (Fig. 5a-c), mimicking effects observed with either constitutively active RhoA or the DAD peptide. In addition, in a co- 
a

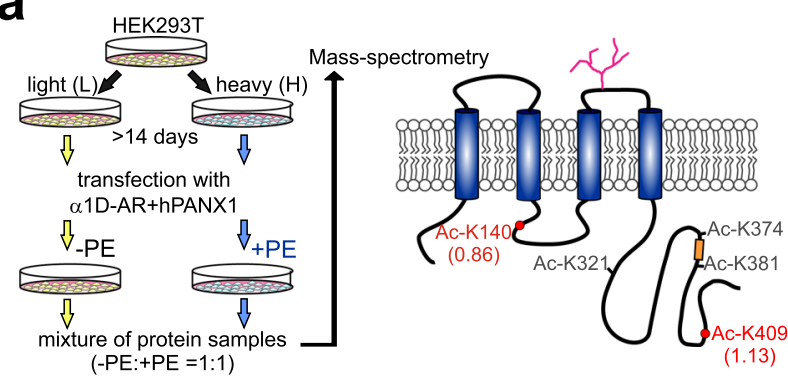

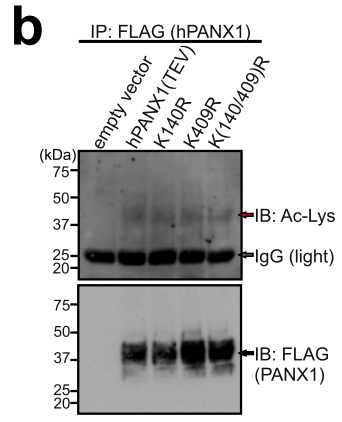

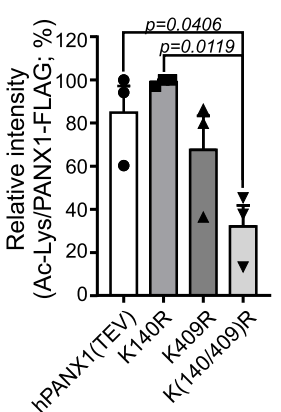

Fig. 6 Identification of acetylated lysine sites on PANX1. a SILAC/MS analysis of hPANX1-Strep immunoprecipitated from control or PE-treated $\alpha 1 D-A R /$ hPANX1-transfected HEK293T whole-cell lysates revealed multiple lysine acetylation sites on hPANX1 (from $n=3$ biological replicates). Abundance levels for the acetylated forms of $\mathrm{K} 140$ or $\mathrm{K} 409$ in the cognate peptides were normalized to the median abundance of PANX1 and calculated as a ratio of PEtreated $(H)$ : vehicle-treated $(L)$ for Lys-140 $(0.86 \pm 0.02 ; 0.88,0.88,0.83)$ and for Lys-409 $(1.13 \pm 0.71 ; 0.92,0.02,2.44)$. Other lysine sites $(K 321, K 374$, or K381) were inconsistently acetylated at baseline across the three samples and/or unaffected by PE treatment. b Example acetyl-lysine blot of immunoprecipitated FLAG-tagged and Arg-substituted hPANX1(TEV) constructs (left); acetyl-lysine signal was first normalized relative to FLAG, and then to peak acetyl-lysine signal for comparisons across experiments (right, $n=3$ independent experiments). Data are presented as mean $\pm s$.e.m. One-way ANOVA $\left(F_{3,8}=6.735, p=0.0140\right)$ with Bonferroni's multiple comparisons test ( $p$ values from comparisons are shown). PE phenylephrine.

immunoprecipitation assay, we found an interaction between coexpressed HDAC6 and PANX1 (Fig. 5d). These data support a hypothesis that HDAC6 may be able to directly activate PANX1 channels.

To test whether HDAC6 can act directly on PANX1 channels in a cell-free system, we excised inside-out membrane patches from HEK293T cells expressing PANX1. As reported previously ${ }^{5}$, we found no basal PANX1 channel activity in this recording configuration under unstimulated conditions. Strikingly, however, when we applied purified HDAC6 to the cytosolic-facing aspect of the patch membrane there was a robust increase in channel activity, which was subsequently inhibited by carbenoxolone (CBX) (Fig. 5e). The HDAC6-activated channels presented with a unitary conductance of $\sim 90 \mathrm{pS}$ (Fig. 5f), as expected for PANX1 channels in inside-out patches ${ }^{5}$. Interestingly, the channels were less 'flickery' with longer open time ( $\sim 8 \mathrm{~ms}$; Fig. 5g) than receptor-activated PANX1 channels recorded in cell-attached configuration ${ }^{5}$, likely reflecting more persistent or complete deacetylation when exposed continuously to HDAC6 in cell-free conditions. These data suggest that a deacetylation mechanism can directly mediate Panxl activation.

To identify potential acetyl-lysine residues on the channel that may be involved in a1D-stimulated Panxl activation, we performed mass spectrometry on Strep-tag-conjugated PANX1 immunoprecipitated from pooled whole-cell samples after stable isotope labeling by amino acids in cell culture (SILAC), with and without PE stimulation (Fig. 6a) ${ }^{39}$. From this analysis $(n=3)$, we tentatively identified several basally acetylated lysine residues on PANX1 (Lys-140, Lys-321, Lys374, Lys-381, and Lys-409; Fig. 6a); among these, the acetylation at Lys-140 and Lys-409 was clearly detected from all three biological replicates, including those peptides with a C-terminally acetylated lysine (i.e., at the trypsin/Lys-C digestion site; Supplementary Fig. 4c-f; Supplementary Table 1). In addition, by acetyl-lysine immunoblot of immunoprecipitated FLAGtagged PANX1, we found that arginine (R) substitutions at Lys140 and Lys-409 resulted in reduced acetylation of the channel (Fig. 6b), consistent with constitutive acetylation on those PANX1 residues.

We next sought functional validation of a role for these particular residues in channel modulation. To test if deacetylation of Lys-140 and/or Lys-409 of PANX1 can evoke channel activation, we recorded from cells expressing two deacetylationmimetic constructs, PANX1(K140R) or PANX1(K409R). Cells expressing PANX1(K140R) presented with large basal whole-cell currents that could not be further activated by $\alpha 1 \mathrm{D}-\mathrm{AR}$ stimulation, whereas PANX1(K409R)-expressing cells had littleto-no basal current and normal PE-induced PANX1 currents (Fig. 7a-c). Notably, whereas Lys-140 is conserved in the mouse Panx1 channel, which can be activated by GaqPCRs (Supplementary Fig. 1a, e, f), Lys-409 is not conserved (i.e., Ala409). These observations suggest a more prominent role for Lys140 than Lys-409 in this channel activation mechanism.

In contrast to the K140R-substituted construct, PANX1 currents were not basally elevated and a1D-AR modulation was intact in cells expressing channels with Gln substitutions at either or both residues (Supplementary Fig. 5). These results with $\mathrm{K} \rightarrow \mathrm{R}$ and $\mathrm{K} \rightarrow \mathrm{Q}$ mutants suggest that constitutive channel activity and occlusion of a1D-AR effects observed with PANX1(K140R) requires the positive charge expected from a deacetylated Lys-140 (and as mimicked by R140). In addition, they also imply that alternative, non-HDAC6 mechanisms may contribute to receptor activation of these Gln-substituted channels. Nevertheless, the large basal PANX1 currents observed in cells expressing either DAD or HDAC6 were not observed with the PANX1-K(140/409) $Q$ construct (Fig. 7d-g), indicating that their deacetylationdependent effects require lysine residues at one or both of these sites. Moreover, basal activity of wild-type PANX1 in HEK293T cells co-expressing wild-type HDAC6 was significantly reduced by tubacin (Fig. $7 \mathrm{~h}$ ), further supporting the hypothesis that the deacetylase activity of HDAC6 is necessary for PANX1 channel activation. Importantly, the HDAC6-mediated increase in PANX1 channel activity in excised inside-out patches was not observed with a PANX1-K(140/409)Q mutant (Fig. 7i); these mutant channels were present and functional since they could be activated by C-terminal cleavage in the same membrane patches (Fig. 7j). Together, these data indicate that HDAC6 can activate PANX1 directly by deacetylation of Lys-140, and that dynamic acetylation-deacetylation of the channel provides a switch mechanism to regulate its activity.

PANX1 channel activation by HDAC6 in $\mathrm{T}$ lymphocytes. T lymphocytes express Panxl channels and various GaqPCRs, including the al-adrenoceptor ${ }^{40}$. We therefore determined effects of al-AR activation on native Panxl channel currents from T cells. PE induced a CBX-sensitive, Panx1-like current in freshly isolated mouse CD4-positive T cells (Fig. 8a, b, Supplementary Fig. 6a) and also activated human PANX1-like currents in a 

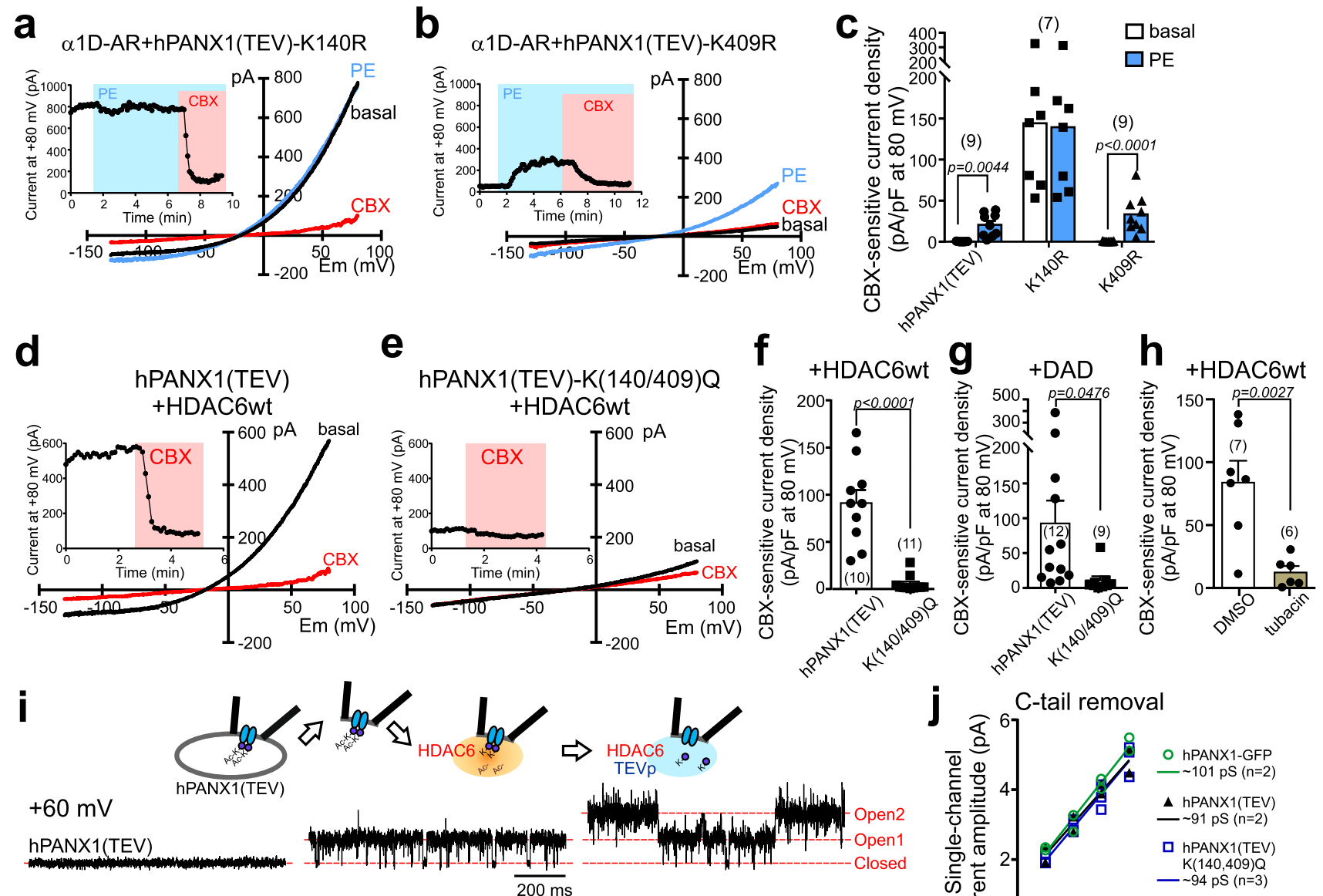

hPANX1(TEV)-K(140/409)Q
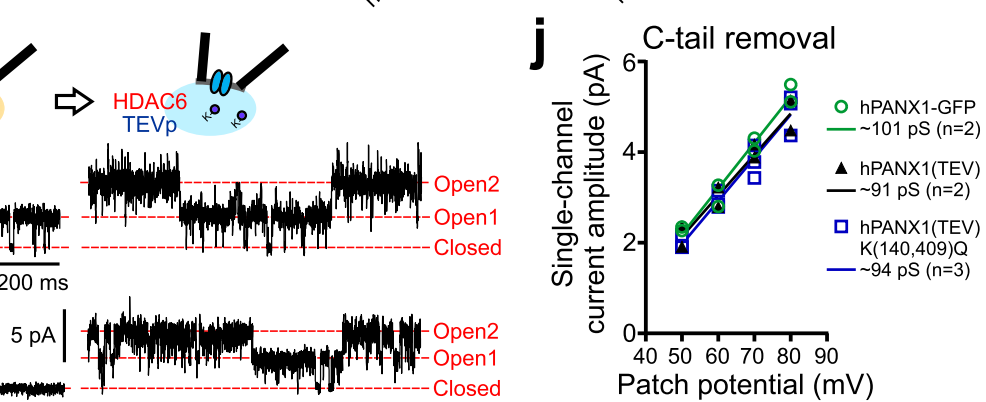

Fig. 7 HDAC6-mediated channel activation by deacetylation of Lys-140 on PANX1. a, b Basal and PE-stimulated whole-cell currents in $\alpha 1 D-A R-$ transfected HEK293T cells expressing the indicated Arg-substituted PANX1 constructs. c Summary data (mean \pm s.e.m) reveal that Arg substitution at Lys140, but not at Lys-409, increased basal PANX1 current and occluded PE effects. $n=9,7$, or 9 cells examined over 3 independent experiments. Two-way ANOVA ( $F_{2,22}=9.924, p=0.0008$ ) with Bonferroni's multiple comparisons test ( $p$ values from comparisons are shown). $\mathbf{d}$, e Effect of HDAC6 on wholecell current in HEK293T cells expressing hPANX1(TEV) or hPANX1(TEV)-K(140/409)Q. $\mathbf{f}, \mathbf{g}$ Summary data reveal that HDAC6 (f) or DAD (g) did not activate basal whole-cell current in cells expressing GIn-substituted hPANX1(TEV) channels. $n=10$ or 11 (f), 12 or 9 (g) cells examined over 6 independent experiments. Data are presented as mean \pm s.e.m. Two-tailed unpaired $t$-test $(\mathbf{f} t=6.425, \mathrm{df}=19 ; \mathbf{g} \mathrm{t}=2.118, \mathrm{df}=19)$. $\mathbf{h}$ Grouped results showing tubacin $(2 \mu \mathrm{M})$, but not DMSO, reduced basal whole-cell currents in cells expressing hPANX1(TEV) channels and wild-type HDAC6 proteins. $n=7$ or 6 cells examined over 3 independent experiments. Data are presented as mean \pm s.e.m. Two-tailed unpaired $t$-test $(t=3.858, d f=11)$. i Inside-out patch recordings of hPANX1(TEV) and hPANX1(TEV)-K(140/409)Q during exposure to HDAC6 and TEV protease. $\mathbf{j}$ Unitary I-Vs for each of the indicated PANX1 channel constructs after activation by C-terminal cleavage with caspase 3 or TEV protease; lines indicate fits by linear regression to estimate the unitary slope conductance $(\gamma)$ for each group (green circles: hPANX1-eGFP, caspase: $\gamma \sim 101$ pS; black triangles: hPANX1(TEV), TEVp: $\gamma \sim 91$ pS; blue squares: hPANX1(TEV)-K(140/409)Q), TEVp: $\gamma \sim 94$ pS; the $n$ values provided are for biologically independent patches). PE phenylephrine, TEVp Tobacco Etch Virus protease.

Jurkat $\mathrm{T}$ cell line (Fig. 8c, d). The PE-evoked current was not observed in Jurkat $\mathrm{T}$ cells after CRISPR/Cas9-mediated deletion of PANX1, indicating that it was indeed due to PANX1 (Supplementary Fig. 6b-d). To test whether HDAC6 can act directly on native PANX1 channels in T cells, we excised membrane patches from Jurkat cells and applied purified HDAC6 to the intracellular side of the membrane. As we had observed with the recombinant PANX1 channels, we found that HDAC6 was also able to activate native PANX1 channels in membrane patches excised from Jurkat cells (Fig. 8e, $n=2$ ); the HDAC6-activated channel presented with a unitary conductance of $\sim 86 \mathrm{pS}$, as expected for PANX1 (Fig. 8f, cf. Fig. 4f). These data indicate that GaqPCRs and HDAC6 activate native Panx1 channels in $\mathrm{T}$ lymphocytes.

\section{Discussion}

PANX1 channels are reversibly activated by multiple Gaqcoupled receptors to mediate release of ATP and support intercellular purinergic signaling ${ }^{15,16,18,41}$. We examined cellular mechanisms by which GaqPCRs activate PANX1 channels, uncovering an unconventional signaling pathway linking the a1D-AR to PANX1 channels via RhoA-mDia-HDAC6, and defining a channel activation mechanism that involves a lysine acetylation-deacetylation switch on PANX1 (Fig. 8g).

Lysine acetylation-deacetylation for ion channel modulation. Protein lysine acetylation is a long-recognized form of reversible posttranslational modification most commonly associated with histones and transcriptional regulation; however, other roles for 
a

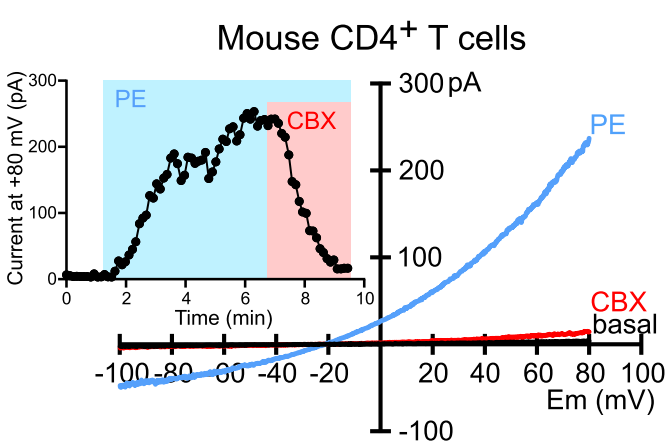

C

e

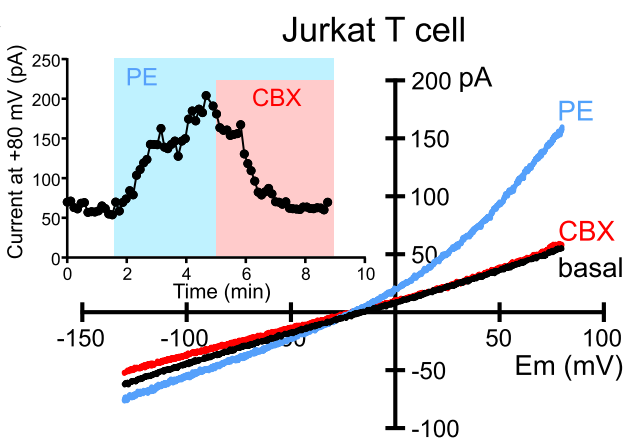

Jurkat T cell b

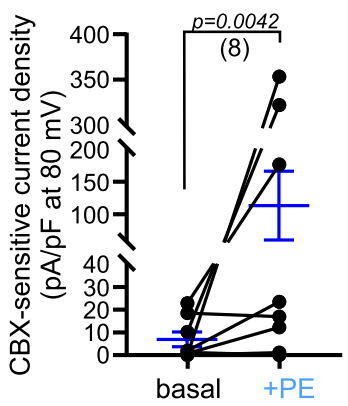

d

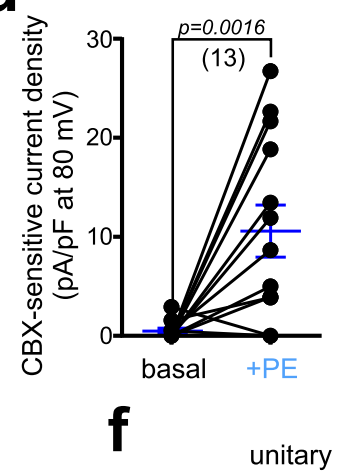

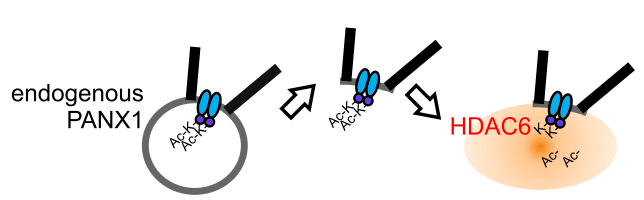

$+60 \mathrm{mV}$

$200 \mathrm{~ms}$

$5 \mathrm{pA}$

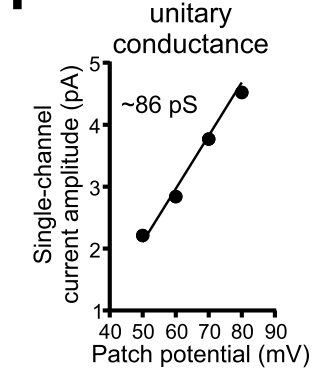

g

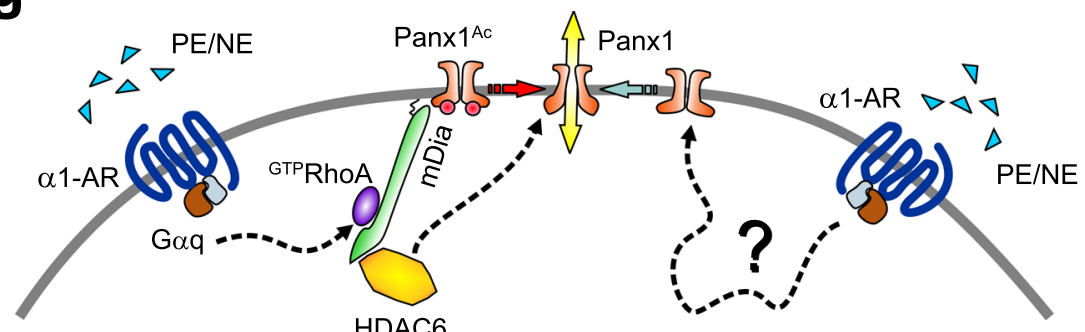

Fig. 8 HDAC6 activates native Panx1 channels in T lymphocytes. a Exemplar recording from a freshly isolated mouse CD4 ${ }^{+} \mathrm{T}$ cell that responded to PE $(20 \mu \mathrm{M})$ with an increase in whole-cell CBX-sensitive current and I-V characteristics of Panx1. b Summary data (mean \pm s.e.m, blue lines) showing CBXsensitive whole-cell current density in mouse CD4 + naïve T cells, before and after bath application of $20 \mu \mathrm{M} P E\left(n=8 ; 5 \mathrm{~T}_{\text {reg }}\right.$ and 3 T eff cells examined over 3 independent experiments); one-tailed paired $t$-test $(t=2.014, \mathrm{df}=7)$. c Representative whole-cell recording from Jurkat $T$ cell that showed increased CBX-sensitive PANX1-like current in response to PE $(20 \mu \mathrm{M})$. d Summary data (mean \pm s.e.m, blue lines) showing CBX-sensitive currents before and after PE stimulation on individual Jurkat T cells; one-tailed paired $t$-test $(n=13$ cells examined over 3 independent experiments; $t=3.682, \mathrm{df}=12)$. e, $\mathbf{f}$ Inside-out recording from a Jurkat T cell showing activation by HDAC6 of a PANX1-like channel (e), with corresponding unitary I-V (f). $\mathbf{g}$ Schematic illustrates the RhoA-mDia-HDAC6 signaling pathway interposed between the Goq-coupled $\alpha 1-A R$ and Panx1 channel activation by lysine deacetylation and alternative pathway(s) not yet defined. PE phenylephrine, NE norepinephrine, CBX carbenoxolone.

protein acetylation are increasingly being recognized ${ }^{36}$. Notably, HDAC6 is found in the cytoplasm where its best known target is tubulin, deacetylation of which can lead to microtubule destabilization and changes in mechanical stress on cell membranes ${ }^{34,35,42}$. Although PANX1 is reported to be a stretchactivated channel $^{9}$, it is unlikely that HDAC6-mediated PANX1 activation was secondary to mechanical effects of tubulin deacetylation, especially in excised patches where such physical forces would be minimal and channel activation by HDAC6 depended on specific lysine residues on PANX1. It is also worth mentioning that HDAC6 modulates various metabolic and signaling enzymes, and lysine deacetylation can be a prelude for protein ubiquitylation and proteasomal degradation ${ }^{35}$. Indeed, the only other known channel regulatory mechanisms associated with direct acetylation-deacetylation involve effects on channel stability (e.g., channel acetylation inhibited ubiquitylation of epithelial $\mathrm{Na}^{+}$ 
channels and Connexin32) 43,44 or membrane localization (e.g., channel deacetylation enhanced membrane trafficking of cardiac $\mathrm{Na}^{+}$channels, $\left.\mathrm{Na}_{\mathrm{V}} 1.5\right)^{45}$. However, we found no effects on membrane PANX1 levels even after extended agonist treatment in cells and, again, those metabolic actions are unlikely to account for increased PANX1 channel activity evoked by HDAC6 in cellfree membrane patches.

Interestingly, the direct PANX1 channel activation by lysine modification that we revealed is loosely analogous to a sumoylation-based regulation first discovered for K2P1 background potassium channels ${ }^{46}$. In that case, previously inactive channels at the cell membrane were unsilenced by enzymatic removal of small ubiquitin-like modifier (SUMO) protein adducts, rather than acetyl groups, from a specific channel lysine residue ${ }^{46}$; however, a signaling pathway that accesses the sumoylation process for $\mathrm{K} 2 \mathrm{P} 1$ channel regulation has not been identified, and $\mathrm{K} 2 \mathrm{P} 1$ activation does not depend on a specific charge at the key lysine residue ${ }^{46}$. By contrast, the lysine deacetylation of PANX1 channels we describe is triggered by a receptor-activated RhoA-mDia-HDAC6 pathway, and channel activation could be mimicked by replacement of Lys-140 with another positively charged residue in PANX1-K140R but not by the neutral substitution obtained with PANX1-K140Q. This suggests that a positive charge at that site (either Arg or deacetylated lysine) may be sufficient for constitutive activation of PANX1 channels. It is important to point out that although Gln substitutions on PANX1 did not induce basal currents and interfered with channel activation by HDAC6, an a1D-activated PANX1 current could still be evoked. This implies that those lysine residues are required for deacetylation-dependent channel activation, but not for an alternative form of GaqPCR-mediated activation (Fig. 8g). It remains to be determined whether and how electrostatic mechanisms supported by Lys-140 might contribute to basal PANX1 channel activation, and what alternative signaling processes support receptor activation of the unacetylated, but basally silent PANX1-K(140/409)Q channels.

Receptor-activated signaling pathways for PANX1 regulation. Our experiments have mapped a mechanism that involves signaling from a1D-AR to PANX1 via RhoA, mDia, and HDAC6. Even as this overall signaling pathway has not been previously delineated, the connections between individual molecular constituents in the pathway have been described. It is well established that p63RhoGEF can transduce Gaq receptor stimulation to RhoA activation ${ }^{21,47}$. Consistent with this, we find that a Gaq construct that does not interact with p63RhoGEF (unlike wildtype Gaq) is unable to rescue PANX1 channel activation in Gaq/ 11-deleted cells. Likewise, $\mathrm{mDia}$ is a well-known downstream effector for RhoA signaling, typically involved in mediating the actin nucleation and elongation associated with Rho-dependent cytoskeletal rearrangements ${ }^{25,27,31}$. In the context of RhoAdependent PANX1 channel activation, a role for $\mathrm{mDia}$ as the relevant RhoA effector was supported by the observation that an F39A variant of the constitutively active RhoA(G14V) construct that couples only to mDia was sufficient for PANX1 channel activation. More limited information links RhoA and mDia to HDAC6, but this was implicated in the context of RhoAmediated disruption of osteoclast maturation that involves $\mathrm{mDia} 2$ activation of HDAC6, and subsequent tubulin deacetylation ${ }^{34}$. In the present work, we provide evidence that PANX1 is itself a substrate for HDAC6, and link this channel modulation to receptor, RhoA and $\mathrm{mDia}$ signaling using pharmacological inhibitors of HDAC6 and mutagenesis of the relevant acetylated lysine residues on PANX1.
Given the widespread importance of purinergic signaling and the ability of activated PANX1 channels to mediate release of ATP and other metabolites, there has been much interest in understanding PANX1 regulation in different contexts. Collectively, these studies have revealed a number of surprising activation mechanisms for this remarkable channel ${ }^{1,48}$. This includes the first example of an irreversible proteolytic activation mechanism by C-terminal caspase cleavage of PANX1 $1^{4,6}$, which has subsequently been observed in other channels ${ }^{49}$. In another example, metabotropic actions by the NMDA receptor channel and the type I TNF receptor appear to regulate PANX1 by a Src family kinase-mediated tyrosine phosphorylation mechanism $29,50,51$. Likewise, our present examination of PANX1 channel activation by Gaq-coupled receptors reveals yet another unexpected signaling pathway, involving RhoA-mDia and an HDAC6-dependent channel deacetylation, and provides additional potential targets for regulating PANX1-dependent intercellular signaling.

Receptor- and HDAC6-mediated regulation of T lymphocytes. We previously showed that PANX1 channels are irreversibly activated in apoptotic $\mathrm{T}$ lymphocytes to release nucleotide findme signals and other metabolites that expedite corpse clearance and support an anti-inflammatory environment ${ }^{4,7}$. Whether GPCR ligands activate PANX1 in T cells has never been explored despite a growing realization that various classical neurotransmitters, including norepinephrine, may serve as modulators of immune cell function ${ }^{20}$. We now provide functional evidence for activation of PANX1 channels by a1-AR signaling in $\mathrm{CD} 4{ }^{+}$ $\mathrm{T}$ cells, raising the possibility that a1-AR-activated PANX1 channels contribute to sympathetic regulation of immune response. A sympathetic neuronal-immune interaction is well recognized in the cholinergic anti-inflammatory pathway in which norepinephrine or epinephrine induces acetylcholine release from $\mathrm{T}$ cells to dampen macrophage activation ${ }^{52-54}$. Whereas those anti-inflammatory effects are attributed primarily to $\beta 2$-adrenoceptors, it was reported that $\alpha 1$-AR signaling in $\mathrm{T}$ cells modulates splenic secretion of interferon $\gamma$ in an arthritis model $^{55}$. In addition, our finding that HDAC6 can directly activate PANX1 channels in $\mathrm{T}$ cells suggests other pathways for immune regulation. For example, HDAC6 and PANX1 are translocated to sites of contact with antigen presenting cells, where they contribute to the organization of immune synapse and $\mathrm{T}$ cell activation ${ }^{56,57}$. Conversely, inhibition of HDAC6 or PANX1 attenuates disease progression in some inflammatory and/or autoimmune mouse models (e.g., arthritis, multiple sclerosis $)^{58-60}$, and HDAC6 negatively regulates activity of $\mathrm{CD} 4^{+}$ Foxp $3^{+}$regulatory $\mathrm{T}$ cells ${ }^{61}$. Our experiments showing activation of PANX1 channels by multiple GaqPCRs, some of which are also found on $\mathrm{T}$ cells and implicated in immune modulation (i.e., 5-HT2, H1, mGluR1 $)^{20}$, suggests the exciting possibility that receptor/HDAC6-activated PANX1 channels could contribute to neurotransmitter-mediated immune modulation.

\footnotetext{
Methods

Animals and reagents. Mice (C57BL6/J) were obtained from Jackson Labs and used following procedures adhering to National Institutes of Health Animal Care and Use Guidelines and approved by the Animal Care and Use Committee of the University of Virginia. Mice were housed in $12 \mathrm{~h}$ light $/ 12 \mathrm{~h}$ dark cycle at room temperature with $40-70 \%$ humidity, with access to food and water ad libitum. All chemicals were purchased from Sigma-Aldrich unless otherwise stated. EZ-linksulfo-NHS-LC-biotin and Fura-2AM were obtained from Thermo Fisher Scientific. Recombinant human HDAC6 was purchased from Millipore. A pro-caspase $3 \Delta 28$ $175 \mathrm{TS}$ deletion mutant in pET-22b $(+)$ (Novagen) was purified from E.coli BL21 (DE3) cells and activated by thrombin cleavage, and Tobacco Etch Virus protease (TEVp, in pRK793) was purified from E. coli BL21(DE3) CodonPlus-RIL cells $(\text { Strategene })^{5}$.
} 
Plasmids and cloning. Plasmid containing mouse adrenergic a1D receptor was obtained from OriGene (MR222643) as previously reported ${ }^{15,62}$. Histamine H1 receptor and metabotropic mGluR1 receptor were obtained from Drs. Graeme Milligan (University of Glasgow) ${ }^{63}$ and Stephen Ikeda (NIAAA, NIH) ${ }^{64}$. Wild-type Gq construct (Gqwt) and GqA253K construct was a gift from Dr. John Tesmer (Purdue University, IN) ${ }^{21}$. C3 exoenzyme and RhoA constructs (T19N, G14V, G14V/T37Y, G14V/F39A) were gifts from Dr. Kodi Ravichandran (University of Virginia, VA ${ }^{23}$. pEGFPm-DAD and pEGFPm-DAD(M1041A) were gifts from Dr.

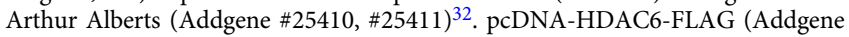
\#30482) and pEGFP-N1-HDAC6cd (catalytic-deficient HDAC6 carrying H216A and H611A mutations; Addgene plasmid \#36189) were gifts from Dr. Tso-Pang $\mathrm{Yao}^{65,66}$. To generate eGFP-tagged wild-type HDAC6, catalytic-deficient HDAC6 was removed from pEGFP-N1-HDAC6cd by enzyme digestion using BglII and HindIII, and was replaced by wild-type HDAC6. The wild-type HDAC6 DNA was amplified by polymerase chain reaction (PCR) from pcDNA-HDAC6-FLAG, using GoPro DNA polymerase (Promega; primers provided in Supplementary Table 2), digested using BglII and HindIII and ligated with T4 DNA ligase (New England Biolabs)

Plasmids encoding mouse Pannexin 1 (mPanx1) and human Pannexin 1 (wildtype hPANX1, hPANX1(TEV), or C-terminally eGFP-tagged hPANX1) were previously described $\mathrm{d}^{5,6,15}$. pFastBac containing C-terminally Strep-tagged hPANX1 was kindly provided by Dr. Mark Yeager (University of Virginia, VA). To generate lysine-to-arginine or lysine-to-glutamine mutations (K140Q, K409Q, K140Q/ K409Q, K140R, and K409R) of human PANX1, site-directed mutagenesis was performed on hPANX1(TEV) construct using Pfu Turbo polymerase (Agilent) and the combinations of primers listed in Supplementary Table 2).

Plasmid encoding Tet-on spCas9 was modified from pCW-Cas $9^{67}$ (provided by Drs. Eric Lander and David Sabatini; Addgene \#50661) to replace the puromycinresistance gene with blasticidin resistance marker. pLX-sgRNA-BfuAI-2k plasmid (Addgene \#112915, a gift from Dr. Ren-Jang Lin) ${ }^{68}$ was engineered to encode two different small guide RNAs (sgRNAs) against human PANX1 gene. First, the blasticidin resistance gene of pLX-sgRNA-BfuAI-2k was replaced by zeocin resistance marker, and one additional U6 promoter was subcloned into this plasmid (pLX-2 $\times$ sgRNA). Complementary oligonucleotides encoding two sgRNAs (sgRNA 1: GCCTTCACCCAGTCACCGGC; sgRNA 2: GATGGTCACGTGCATTGCGGT) were subsequently inserted into pLX $-2 \times$ sgRNA using T4 DNA ligase at BsbI and BsmMI sites.

All constructs were verified by DNA sequencing.

Mammalian cell culture and transfection. HEK293T cells (passage 7-20, ATCC), embryonic fibroblasts (Fq11 cells) derived from Gq/11 knockout mice ${ }^{69}$, and Gq/ $11 / 12 / 13$-deleted HEK293 cells ${ }^{65,66}$ were cultured at $37^{\circ} \mathrm{C}$ with humidified air containing $5 \% \mathrm{CO}_{2}$ in Dulbecco's Modified Eagle Medium (DMEM, high glucose, Gibco) containing $10 \%$ fetal bovine serum (FBS, Gibco), penicillin, streptomycin, and sodium pyruvate. Fq11 cells were kindly provided by Dr. Patricia Hinkle (University of Rochester, Rochester, NY) ${ }^{69}$, and Gq/11/12/13-deleted HEK293 cell $^{70}$ was a generously gift from Dr. Asuka Inoue (Tohoku University, Miyagi, Japan). Jurkat T cells (E6.1, ATCC) were maintained in RPMI 1640 (Corning) with $10 \%$ FBS, penicillin, streptomycin, and L-glutamine at a density of $0.5-1.5$ cells ml ${ }^{-1}$. Transfections were carried out by using either Lipofectamine2000 ${ }^{\text {Dxt }}$ (Invitrogen) or Transporter 5 (Polysciences) following manufacturers' manuals.

\section{Deletion of PANX1 from Cas9 stably-expressed Jurkat T cell line. For pro-} duction of lentivirus expressing Tet-on spCas9, HEK293T cells were seeded in 10 $\mathrm{cm}$ dish, and transfected the following day with pMD2.G and psPAX packaging vectors (Addgene \#12259, Addgene\#12260; both from Dr. Didier Trono), along with the targeting construct encoding Tet-on spCas9, using FuGENE-6 (Promega). Media was refreshed $6 \mathrm{~h}$ after transfection. Viral supernatants were collected $48 \mathrm{~h}$ and $72 \mathrm{~h}$ after first media refreshment, flash-frozen with liquid nitrogen, and stored

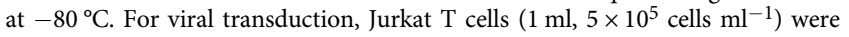
incubated with $3 \mathrm{ml}$ viral supernatant in a six-well plate with protamine sulfate $(10$ $\mu \mathrm{g} \mathrm{ml}^{-1}$; from $10 \mathrm{mg} \mathrm{ml}^{-1}$ stock). This mixture was spun $1000 \times g$ at $32^{\circ} \mathrm{C}$ for $2 \mathrm{~h}$; this procedure was repeated the following day. After $24 \mathrm{~h}$, virally transduced Jurkat $\mathrm{T}$ cells were cultured in media containing blasticidin $\left(15 \mu \mathrm{g} \mathrm{ml}{ }^{-1}\right)$ and zeocin $(200$ $\mu \mathrm{g} \mathrm{ml}^{-1}$ ) for 3 and 5 days, respectively. Virally transduced Jurkat T cells were treated with doxycycline $(50 \mu \mathrm{M})$ for $48 \mathrm{~h}$ to induce expression of spCas 9 . After 48 $\mathrm{h}$ of induction, $5-10 \times 10^{6}$ cells were washed and resuspended in $400 \mu \mathrm{l}$ plain RPMI 1640, followed by electroporation at $250 \mathrm{mV}$ for $25 \mathrm{~ms}$ per pulse (BTX Electro Square Porator T820, Harvard Apparatus) with $10 \mu \mathrm{g}$ of plasmids containing two PANX1 sgRNAs. Electroporated cells were further incubated in doxycycline for another $48 \mathrm{~h}$, and then were cultured in selection media containing zeocin $(400 \mu \mathrm{g}$ $\mathrm{mL}^{-1}$ ). After 1 week, cells were serially diluted into 96-well plates to generate oligo clones and then transferred to larger wells for continued expansion.

Isolation of mouse primary CD4 ${ }^{+} \mathbf{T}$ cells. Naïve $\mathrm{CD}^{+} \mathrm{T}$ cells were isolated from spleens of wild-type C57BL/6 J mice (8-14 weeks; both sexes). In brief, dissected mouse spleens were gently ground through $70 \mu \mathrm{m}$ cell strainer mesh (Falcon), and naïve $\mathrm{CD}^{+} / \mathrm{CD}^{2} 5^{+}$(regulatory; $\mathrm{T}_{\text {reg }}$ ) or $\mathrm{CD}^{+} / \mathrm{CD}^{2} 5^{-}$(effector; $\mathrm{T}_{\text {eff }}$ ) $\mathrm{T}$ cells were isolated and collected using a CD $4^{+} \mathrm{CD} 25^{+} \mathrm{T}$ cell isolation kit (Miltenyi Biotec;
\#130-091-041) and magnetic separation columns (Miltenyi Biotec, \#130-042-201 and \#130-042-401) according to manufacturer's protocol. Purity of T cell populations were verified by using flow cytometry (Supplementary Fig. 6a; CD3-PerCP Cy5.5 1:100, eBioscience, \#45-0031-80; CD4-Pacific Blue 1:100, eBioscience, \#570042-82; and Foxp3-APC 1:100, eBioscience, \#17-5773-80; FlowJo v.10.6.2). Isolated $\mathrm{CD}^{+} \mathrm{T}$ cells were resuspended in RPMI 1640 containing 10\% FBS, 1\% PSQ, $1 \%$ sodium pyruvate, $1 \%$ HEPES, and $1 \%$ nonessential amino acids, and plated on poly-L-lysine coated coverslips $>1 \mathrm{~h}$ before recording. Whole-cell recording of CD4 $+\mathrm{T}$ cells was carried out within $48 \mathrm{~h}$ after isolation.

Electrophysiology. All voltage-clamp recordings were carried out at room temperature, using an Axopatch 200B amplifier controlled by pCLAMP10 software and digitized using Digidata 1322 A digitizer (all Molecular Devices). Micropipettes were pulled from thin-walled, fire-polished borosilicate glass capillaries (Harvard Apparatus) using a P-97 Flaming/Brown Micropipette Puller (Sutter Instrument), and coated with Sylgard 184 silicone elastomer (Dow Corning Corporation).

Cell-attached patch recordings were performed using micropipettes with resistance of 5-10 $\mathrm{M} \Omega$, and filtered to $1 \mathrm{kHz}$ using the Axopatch 200B. Bath and pipette solution contained $140 \mathrm{mM} \mathrm{NaCl}, 3 \mathrm{mM} \mathrm{KCl}, 2 \mathrm{mM} \mathrm{MgCl}, 2 \mathrm{mM} \mathrm{CaCl}_{2}$ $10 \mathrm{mM}$ HEPES, and $10 \mathrm{mM}$ glucose (pH 7.3; 300 mOsm). After $\geq 10 \mathrm{G} \Omega$ seals were obtained, cells were exposed to bath solution containing phenylephrine ( $\mathrm{PE}$ $20 \mu \mathrm{M})$. After steady-state channel activity was acquired (at $+90 \mathrm{mV})$, the bath solution was further exchanged for one containing both PE $(20 \mu \mathrm{M})$ and carbenoxolone (CBX, $50 \mu \mathrm{M})$. Maximum Po is derived from NPo divided by the maximum numbers of channel observed in a given patch.

Whole-cell recordings were obtained in the same HEPES-based bath solution (above) using 3-5 $\mathrm{M} \Omega$ borosilicate glass patch pipettes, filled with internal (pipette) solution composed of: $100 \mathrm{mM} \mathrm{CsMeSO}_{4}, 30 \mathrm{mM} \mathrm{TEACl}, 4 \mathrm{mM} \mathrm{NaCl}, 1 \mathrm{mM}$ $\mathrm{MgCl}_{2}, 0.5 \mathrm{mM} \mathrm{CaCl}_{2}, 10 \mathrm{mM}$ HEPES, $10 \mathrm{mM}$ EGTA, $3 \mathrm{mM}$ ATP-Mg, and 0.3 $\mathrm{mM}$ GTP-Tris ( $\mathrm{pH} 7.3 ; \sim 290 \mathrm{mOsm}$ ). In some experiments, intracellular free calcium was buffered to $10 \mathrm{nM}$ by replacing EGTA with BAPTA (tetrapotassium salt, $14.3 \mathrm{mM}$ ), or GTP-Tris was replaced with GTP-lithium or GDP $\beta$-lithium (also $0.3 \mathrm{mM}$ ). Ramp voltage-clamp commands were applied at 7 -s intervals, with voltage ranging from -130 to $80 \mathrm{mV}(0.2 \mathrm{mV} / \mathrm{ms})$ in HEK293T and Jurkat T cells or from -100 to $80 \mathrm{mV}(0.26 \mathrm{mV} / \mathrm{ms})$ in mouse $\mathrm{CD} 4^{+} \mathrm{T}$ cells. CBX-sensitive current was taken as the difference in current at $+80 \mathrm{mV}$ before and after CBX application, and was normalized to cell capacitance (i.e., current density). PEinduced current was determined by the difference in CBX-sensitive current at +80 $\mathrm{mV}$, before and after PE application in bath. To ensure that recorded cells coexpressed signaling proteins (e.g., RhoA(T19N) or C3 exoenzyme) along with a1DARs and mPanxl channels, we transfected cells with a DNA mixture that included (from highest to lowest concentration) alD-AR, signaling protein constructs, mPanx1 channel, and a GFP-containing vector. Thus, co-transfected cells were identified by expression of GFP and presence of basal outwardly rectifying currents that were sensitive to CBX (i.e., Panxl currents), the components that were transfected at the lowest DNA concentrations.

Inside-out patch recording were obtained using micropipettes with resistance of 5-10 M $\Omega$; data were filtered to $5 \mathrm{kHz}$ using an 8-pole low-pass Bessel filter (LPF-8, Warner Instruments) and digitized at $20 \mathrm{kHz}$. Pipettes were filled with HEPES based bath solution (above) and, after seal formation $(\geq 10 \mathrm{G} \Omega$ ), the membrane patch was excised and the bath solution was exchanged for an inside-out solution composed of: $150 \mathrm{mM} \mathrm{CsCl}, 5 \mathrm{mM}$ EGTA, $10 \mathrm{mM}$ HEPES, and $1 \mathrm{mM} \mathrm{MgCl} 2(\mathrm{pH}$ 7.3). Patches were held at +50 to $+80 \mathrm{mV}(\Delta 10 \mathrm{mV})$. We excluded membrane patches that displayed non-Panx1 channel activity, as determined by lack of CBX sensitivity and unitary channel properties unlike Panx 1 channels ${ }^{5}$. As previously reported, we did not observe basally-active Panx1 channels immediately following patch excision ${ }^{5}$. Recombinant human HDAC6 (Millipore) was applied near the patch under stop-flow conditions to a final concentration of $\sim 1 \mu \mathrm{g} \mathrm{ml}{ }^{-1}$. Whenever possible, after steady-state channel activity was obtained (usually within $8 \mathrm{~min}$ after HDAC6 application), activated caspase 3 or TEV protease was applied $(\sim 1-2 \mu \mathrm{g} \mathrm{ml}$ ${ }^{-1}$ ) and the steady-state channel activity was recorded ${ }^{5}$. Single-channel recordings were analyzed using pCLAMP10; channel data were filtered to $2 \mathrm{kHz}$ using an 8 pole low-pass Bessel filter for analysis of open probability, and filtered to $1 \mathrm{kHz}$ for presentation.

Cell-surface biotinylation and immunoprecipitation. Cell-surface biotinylation were carried out in HEK293T cells that were transiently transfected with wild-type PANX1 and FLAG-tagged $\alpha 1 \mathrm{D}-\mathrm{AR}$ for $\sim 24 \mathrm{~h}$ using Transporter ${ }^{\mathrm{m}} 5$ (Polysciences). Transfected cells were washed and incubated in HEPES-based bath solution (above) for $15 \mathrm{~min}$ at room temperature, and subsequently incubated with the same buffer containing $20 \mu \mathrm{M}$ PE for additional $0 \sim 20 \mathrm{~min}(\Delta 5 \mathrm{~min}$ ). After PE stimulation, these cells were washed with cold phosphate-buffered saline (PBS, Gibco) and incubated in cold PBS containing EZ-Link Sulfo-NHS-Biotin ${ }^{\text {tx }}\left(1 \mathrm{mg} \mathrm{ml}^{-1}\right.$; Thermo Scientific) for $>1 \mathrm{~h}$ at $4{ }^{\circ} \mathrm{C}$ with gentle rocking. After biotin-containing PBS was removed, cells were further incubated with PBS containing $100 \mathrm{mM}$ glycine at $4^{\circ} \mathrm{C}$ for $>30$ min to quench the reaction. Cells were lysed in PBS buffer containing $1 \%$ Triton X-100, protease inhibitor cocktail (Sigma-Aldrich; P8340), $10 \mathrm{mM} \mathrm{NaF}$ and $10 \mathrm{mM} \mathrm{NaVO}$ (lysis buffer). Protein samples were mixed with streptavidinagarose beads (Thermo Scientific) for $>2 \mathrm{~h}$ at $4^{\circ} \mathrm{C}$ to collect biotinylated proteins. 
To determine constitutive lysine acetylation on PANX1, C-terminally FLAGtagged hPANX1(TEV) constructs, with or without mutation of Lys-140 or Lys-409, were transiently transfected in HEK293T cells using Transporter ${ }^{\text {sin }}$ 5. Cells were incubated with $2 \mu \mathrm{M}$ tubacin after transfection ( $24 \mathrm{hr}$ ), transferred to lysis buffer containing $5 \mu \mathrm{M}$ tubacin and $2 \mu \mathrm{M}$ trichostatin A (TSA). Protein samples were mixed with anti-FLAG M2 agarose beads (Sigma-Aldrich, \#A2220) for $>2 \mathrm{~h}$ at $4{ }^{\circ}$ C, with constant shaking.

To examine protein-protein interaction between PANX1 and HDAC6, HEK293T cells were transiently transfected with Strep-tagged PANX1 and FLAGtagged HDAC6 for $\sim 24 \mathrm{~h}$ using Transporter ${ }^{\mathrm{m} x} 5$. Cells were lysed and protein samples were prepared as mentioned above. Anti-FLAG M2 affinity agarose gel (Sigma-Aldrich) was used to pull down HDAC6-FLAG proteins.

After extensive wash in lysis buffer, protein samples were mixed with $5 \times$ Laemmli buffer (62.5\% glycerol, $12.5 \%$ SDS, $0.5 \%$ bromophenol blue, $25 \%$ fresh 2 mercaptoenthanol in $30 \mathrm{mM}$ Tris- $\mathrm{HCl}, \mathrm{pH}$ 6.8) at room temperature for $5 \mathrm{~min}$, and separated from beads using microcentrifuge spin columns (Thermo Scientific).

Immunoblotting. Protein samples were separated by SDS-PAGE, transferred onto $0.45 \mu \mathrm{m}$ nitrocellulose membranes (PerkinElmer) and blocked with $5 \%$ non-fat dry milk dissolved in a Tris-based buffer $(10 \mathrm{mM}$ Tris, $150 \mathrm{mM} \mathrm{NaCl}$, and $0.1 \%$ Tween $20, \mathrm{pH}$ 7.4) at room temperature for $1 \mathrm{~h}$. Human PANX1 proteins were detected by incubating with anti-Panx1 antibody (Cell Signaling Biotechnology; \#91137; 1:1000). Anti-FLAG M2 (Sigma-Aldrich, \#F3156; 1:1000) was used to detect Mycand FLAG-tagged alD-AR proteins or FLAG-tagged HDAC6. As a loading control for cell-surface biotinylation samples, we used an antibody to $\mathrm{Na}^{+} / \mathrm{K}^{+}$-ATPase (Cell Signaling, \#3010; 1:1000). Anti-acetylated lysine antibody (Cell Signaling, $\# 9441$; 1:1000) was used to probe lysine acetylation on hPANX1 proteins. Anti- $\beta$ actin-peroxidase antibody (Sigma-Aldrich; \#A3854; 1:50,000) was used as a loading control. Amersham ECL horseradish peroxidase (HRP)-linked secondary antibodies (GE Healthcare; anti-Rabbit IgG: NA9340V or anti-Mouse IgG: NA931V; 1:6000-10,000) and Western Lightning Plus ECL were used to visualize immunoreactive signals on Amersham Hyperfilm ECL (GE Healthcare). Acetylated lysine signal was imaged by using ChemiDoc ${ }^{\text {mox }}$ Imaging Systems (BioRad). Images of co-immunoprecipitated HDAC6-FLAG and PANX1-Strep were taken by using ImageQuant LAS4000 Mini (GE Healthcare). Quantification of immunoreactive signal was performed by using ImageJ software (v1.8; NIH).

\section{Measurement of intracellular release of calcium. Gaq/11/12/13-deleted} HEK293 cells ${ }^{70}$ were transiently transfected with a1D-AR and Gaq constructs (Gaqwt or GaqA253K) using Lipofectamine 2000 as described above. Forty-eight hours after transfection, cells were trypsinized, centrifuged and resuspended in Ringer solution ( $155 \mathrm{mM} \mathrm{NaCl}, 4.5 \mathrm{mM} \mathrm{KCl}, 1 \mathrm{mM} \mathrm{MgCl} 2,5 \mathrm{mM}$ HEPES, $10 \mathrm{mM}$ D-glucose, $2 \mathrm{mM} \mathrm{CaCl}_{2}$ ) containing $5 \mu \mathrm{M}$ Fura-2AM, $500 \mu \mathrm{M}$ probenecid, $0.02 \%$ Pluronic F-127 (Invitrogen, P3000MP) covered from light. After 30 min incubation at room temperature, Fura-2AM-loaded cells were resuspended in DMEM containing $10 \% \mathrm{FBS}, 500 \mu \mathrm{M}$ probenecid, and $1.5 \times 10^{5}$ cells were plated in each well of 96-well plates (Cellvis, P96-1-N; poly-lysine-coated) and kept at room temperature for additional 20 min covered from light. FlexStation 3 (Molecular Devices) was used to detect fluorescence intensity using Softmax Pro 7 software (excitation wavelengths: $340 \mathrm{~nm} / 380 \mathrm{~nm}$; emission: $510 \mathrm{~nm}$ ). Fura-2AM-loaded cells were first incubated in calcium-free Hank's buffered salt solution $(155 \mathrm{mM} \mathrm{NaCl}, 4.5 \mathrm{mM}$ $\mathrm{KCl}, 1 \mathrm{mM} \mathrm{MgCl}$, $5 \mathrm{mM}$ HEPES, $10 \mathrm{mM}$ D-glucose, $0.5 \mathrm{mM} \mathrm{EDTA}$ ) for $2 \mathrm{~min}$ to obtain baseline fluorescence intensity, followed by application of $20 \mu \mathrm{M}$ PE or $1 \mu \mathrm{M}$ thapsigargin. The peak intensity of PE- or thapsigargin-triggered release of intracellular calcium was determined by averaging at least three independent measurements. Subsequently, extracellular free calcium $(2 \mathrm{mM})$ and ionomycin $(5 \mu \mathrm{M})$ were applied sequentially (as controls) to determine the amplitude of storeoperated $\mathrm{Ca}^{2+}$ entry and the maximum intensity of $\mathrm{Ca}^{2+}$-dependent fluorescence in the cells.

Stable isotope labeling with amino acids in cell culture. HEK293T cells were cultured in DMEM containing $10 \% \mathrm{FBS}$, with either L-lysine-2HCl/L-arginine- $\mathrm{HCl}$ (light labeled) or ${ }^{13} \mathrm{C}_{6}{ }^{15} \mathrm{~N}_{2}$ L-lysine-2 $\mathrm{HCl} /{ }^{13} \mathrm{C}_{6}{ }^{15} \mathrm{~N}_{4} \mathrm{~L}$-arginine $\mathrm{HCl}$ (heavy labeled) (Thermo Fisher Scientific, A33972) for $>14$ days at $37^{\circ} \mathrm{C}$ with humidified air containing $5 \% \mathrm{CO}_{2}$. To identify potential lysine acetylation residues of PANX1, differentially labeled cells were separately transfected with a1D-AR and Strep-tagconjugated hPANX1 using Transporter ${ }^{m} 5$ (Polysciences). Twenty-four hours after transfection, both groups of cells were washed once with HEPES-based bath solution (above) and then incubated in that solution for additional $15 \mathrm{~min}$ at room temperature. Heavy-labeled cells were then further incubated in bath solution containing $20 \mu \mathrm{M}$ PE, whereas light-labeled cells were separately incubated in bath solution alone, both at room temperature for $10 \mathrm{~min}$.

Two groups (heavy labeled and light labeled) of cells were independently lysed using a lysis buffer composed of $150 \mathrm{mM} \mathrm{NaCl}, 50 \mathrm{mM}$ Tris- $\mathrm{HCl}, 0.5 \%$ CHAPS, 5 mM EDTA, Halt ${ }^{\mathrm{m}}$ protease and phosphatase inhibitor cocktail (Thermo Scientific; \#78440), $5 \mu \mathrm{M}$ trichostatin $\mathrm{A}$, and $5 \mu \mathrm{M}$ tubacin. Equal amounts of two protein samples were pooled and incubated with Strep-Tactin Superflow Plus beads
(Qiagen; \#30002) at $4{ }^{\circ} \mathrm{C}$ with constant rocking for $>2 \mathrm{~h}$. Precipitated protein samples were washed twice using Wash Buffer A $(250 \mathrm{mM} \mathrm{NaCl}, 50 \mathrm{mM}$ Tris- $\mathrm{HCl}$, and $0.1 \%$ CHAPS, $\mathrm{pH}$ 7.4), followed by two washes with Wash Buffer B (150 mM $\mathrm{NaCl}, 50 \mathrm{mM}$ Tris- $\mathrm{HCl}$ and $0.1 \%$ CHAPS, $\mathrm{pH}$ 7.4). Protein samples were reconstituted in GlycoBuffer 2 (New England BioLabs), and mixed with $5 \mu \mathrm{M}$ trichostatin A, $5 \mu \mathrm{M}$ tubacin, and Halt ${ }^{\mathrm{m}}$ protease and phosphatase inhibitor cocktail along with PNGase F (New England Biolabs; \#P0708; 40 Unit $\mu \mathrm{L}-1$ ) to remove glycosylation of hPANX1 proteins. After $>2 \mathrm{~h}$ incubation at room temperature, samples were washed twice using Wash Buffer C $(500 \mathrm{mM} \mathrm{NaCl}, 50 \mathrm{mM}$ Tris- $\mathrm{HCl}$ and $0.4 \%$ CHAPS, pH 7.4), followed by two further washes with Wash Buffer B. All wash steps were done at $4{ }^{\circ} \mathrm{C}$ with constant rocking.

To collect hPANX1 proteins with or without PE stimulation, precipitated proteins were eluted from Strep-Tactin beads by using Wash Buffer A containing $10 \mathrm{mM}$ desthiobiotin, collected by using microcentrifuge spin columns, and concentrated by using Amicon Ultra $0.5 \mathrm{~mL}$ filter unit (Millipore Sigma; $30 \mathrm{KDa}$ cutoff). Concentrated protein samples were boiled in $6 \times$ Laemmli buffer for $>5$ min, followed by SDS-PAGE separation using Mini-PROTEAN TGX ${ }^{m}$ precast gels (BioRad; 10\%). Colloidal blue staining (Invitrogen; \#LC6025) was performed to visualize precipitated protein samples prior to mass spectrometry analysis.

Mass spectrometry. Colloidal blue-stained gel bands were destained in $25 \mathrm{mM}$ $\mathrm{NH}_{4} \mathrm{HCO}_{3}, 50 \%$ acetonitrile, $\mathrm{pH} 8.3$. After vacuum drying, the gel bands were digested with $100 \mu \mathrm{L}$ of trypsin/Lys-C (Promega) $(20 \mu \mathrm{g} \mathrm{mL}-1$ in $25 \mathrm{mM}$ $\mathrm{NH}_{4} \mathrm{HCO}_{3}$ ) at $37^{\circ} \mathrm{C}$, overnight. The digested peptides were extracted with $100 \mu \mathrm{L}$ $70 \%$ acetonitrile, $5 \%$ formic acid. Each peptide sample was desalted (ZipTips, EMD Millipore), dried by vacuum centrifugation, and resuspended in $16 \mu \mathrm{L} 0.1 \%$ TFA Samples were loaded on a C18 nanobore trap column (Acclaim PepMap100 C18, 2 $\mathrm{cm}$, nanoViper, Thermo Scientific), and resolved on a C18 Easy-Spray column (Acclaim PepMap RSLC C18, $2 \mu \mathrm{m}, 100 \AA, 75 \mu \mathrm{m} \times 500 \mathrm{~mm}$, nanoViper, Thermo Scientific) by nanoflow LC (EASY-nLC 1200, Thermo Fisher Scientific) coupled online with an Orbitrap Fusion Lumos Tribrid MS (Thermo Fisher Scientific). The peptides were resolved using a linear gradient of $2 \%$ mobile phase B (95\% acetonitrile with $0.1 \%$ formic acid) to $32 \%$ mobile phase B within $60 \mathrm{~min}$ at a constant flow rate of $250 \mathrm{~nL} \mathrm{~min}{ }^{-1}$. The $\mathrm{C} 18$ Easy-Spray column was heated at $50{ }^{\circ} \mathrm{C}$ during the analysis. The 12 most intense molecular ions in each MS scan were sequentially selected for high-energy collisional dissociation $(\mathrm{HCD})$ using a normalized collision energy of $35 \%$. The mass spectra were acquired at the mass range of $\mathrm{m} / \mathrm{z}$ $400-1600$. The Easy-Spray Ion Source (Thermo Scientific) capillary voltage and temperature were set at $2.0 \mathrm{kV}$ and $275^{\circ} \mathrm{C}$, respectively. Dynamic exclusion $(15 \mathrm{~s})$ was enabled to minimize redundant peptide fragmentation events. The RF lens was set to 30\% during the MS analysis and both MS1 and MS2 spectra were collected in profile mode. Data were searched against a Swiss-Prot human protein database (http://www.uniprot.org/uniprot/) using Proteome Discoverer (v.2.1.1.21, Thermo Fisher Scientific) via Mascot (v. 2.5.1, Matrix Science Inc.) with the automatic decoy search option set followed by false-discovery rate (FDR) processing by Percolator (v.3.5). The exact Panx1 (Q96RD7) protein sequence used in the experiments (including the engineered tag and spacer) was added to the Swiss-Prot human protein database. Data were searched with a precursor mass tolerance of 10 ppm and a fragment ion tolerance of $0.05 \mathrm{Da}$, a maximum of two tryptic miscleavages and dynamic modifications for oxidation (15.9949 Da) on methionine residues and for acetylation $(42.01056 \mathrm{Da})$ on lysine residues. The heavy SILAC labeled lysine $(8.014199 \mathrm{Da})$ and arginine $(10.008269 \mathrm{Da})$ residues were also set for dynamic modification. Resulting peptide spectral matches (PSMs) were filtered using an FDR of $\leq 1 \%$ (Percolator $\mathrm{q}$-value $\leq 0.01$ ). Although trypsin may cleave less efficiently at acetylated lysine residues, we included MS identification of peptides with C-terminally acetylated lysines (i.e., at the trypsin/Lys-C cleavage site) since the modified Lys residues were clearly defined by their spectral characteristics and were observed in all three analyses. Spectrum profile is available at Proteomics Identification Database (PRIDE; Project accession: PXD025912).

Statistics. Data are presented as mean \pm s.e.m. Statistical analyses, described in figure legends, were performed using GraphPad Prism (v.9).

Reporting summary. Further information on research design is available in the Nature Research Reporting Summary linked to this article.

\section{Data availability}

All data generated in this study are provided in the Supplementary Information/Source Data file. The raw mass spectrometry proteomics data have been deposited to the ProteomeXchange Consortium via the $\mathrm{PRIDE}^{71}$ partner repository with the dataset identifier PXD025912. Source data are provided with this paper.

Received: 8 June 2019; Accepted: 12 July 2021;

Published online: 23 July 2021 


\section{References}

1. Chiu, Y. H., Schappe, M. S., Desai, B. N. \& Bayliss, D. A. Revisiting multimodal activation and channel properties of Pannexin 1. J. Gen. Physiol. 150, 19-39 (2018).

2. Burnstock, G. \& Knight, G. E. Cell culture: complications due to mechanical release of ATP and activation of purinoceptors. Cell Tissue Res. 370, 1-11 (2017).

3. Penuela, S., Harland, L., Simek, J. \& Laird, D. W. Pannexin channels and their links to human disease. Biochem. J. 461, 371-381 (2014).

4. Chekeni, F. B. et al. Pannexin 1 channels mediate 'find-me' signal release and membrane permeability during apoptosis. Nature 467, 863-867 (2010).

5. Chiu, Y. H. et al. A quantized mechanism for activation of pannexin channels. Nat. Commun. 8, 14324 (2017).

6. Sandilos, J. K. et al. Pannexin 1, an ATP release channel, is activated by caspase cleavage of its pore-associated C-terminal autoinhibitory region. J. Biol. Chem. 287, 11303-11311 (2012).

7. Medina, C. B. et al. Metabolites released from apoptotic cells act as tissue messengers. Nature 580, 130-135 (2020).

8. Furlow, P. W. et al. Mechanosensitive pannexin-1 channels mediate microvascular metastatic cell survival. Nat. Cell Biol. 17, 943-952 (2015).

9. Bao, L., Locovei, S. \& Dahl, G. Pannexin membrane channels are mechanosensitive conduits for ATP. FEBS Lett. 572, 65-68 (2004).

10. Silverman, W. R. et al. The pannexin 1 channel activates the inflammasome in neurons and astrocytes. J. Biol. Chem. 284, 18143-18151 (2009).

11. Wang, J. et al. The membrane protein Pannexin1 forms two open-channel conformations depending on the mode of activation. Sci. Signal. 7, ra69 (2014).

12. Santiago, M. F. et al. Targeting pannexin 1 improves seizure outcome. PLoS ONE 6, e25178 (2011).

13. Kienitz, M. C., Bender, K., Dermietzel, R., Pott, L. \& Zoidl, G. Pannexin 1 constitutes the large conductance cation channel of cardiac myocytes. J. Biol. Chem. 286, 290-298 (2011).

14. Seminario-Vidal, L. et al. Thrombin promotes release of ATP from lung epithelial cells through coordinated activation of rho- and $\mathrm{Ca} 2+-$ dependent signaling pathways. J. Biol. Chem. 284, 20638-20648 (2009).

15. Billaud, M. et al. A molecular signature in the pannexin1 intracellular loop confers channel activation by the alphal adrenoreceptor in smooth muscle cells. Sci. Signal. 8, ra17 (2015)

16. Billaud, $M$. et al. Pannexin1 regulates alpha1-adrenergic receptor- mediated vasoconstriction. Circ. Res. 109, 80-85 (2011).

17. Weaver, J. L. et al. Hematopoietic pannexin 1 function is critical for neuropathic pain. Sci. Rep. 7, 42550 (2017).

18. Locovei, S., Wang, J. \& Dahl, G. Activation of pannexin 1 channels by ATP through P2Y receptors and by cytoplasmic calcium. FEBS Lett. 580, 239-244 (2006).

19. DeLalio, L. J. et al. Constitutive SRC-mediated phosphorylation of pannexin 1 at tyrosine 198 occurs at the plasma membrane. J. Biol. Chem. 294, 6940-6956 (2019).

20. Franco, R., Pacheco, R., Lluis, C., Ahern, G. P. \& O’Connell, P. J. The emergence of neurotransmitters as immune modulators. Trends Immunol. 28, 400-407 (2007).

21. Shankaranarayanan, A. et al. Galpha $\mathrm{q}$ allosterically activates and relieves autoinhibition of p63RhoGEF. Cell Signal. 22, 1114-1123 (2010).

22. Aktories, K. \& Hall, A. Botulinum ADP-ribosyltransferase C3: a new tool to study low molecular weight GTP-binding proteins. Trends Pharmacol. Sci. 10, 415-418 (1989).

23. Tosello-Trampont, A. C., Nakada-Tsukui, K. \& Ravichandran, K. S. Engulfment of apoptotic cells is negatively regulated by Rho-mediated signaling. J. Biol. Chem. 278, 49911-49919 (2003).

24. Seminario-Vidal, L. et al. Rho signaling regulates pannexin 1-mediated ATP release from airway epithelia. J. Biol. Chem. 286, 26277-26286 (2011).

25. Palazzo, A. F., Cook, T. A., Alberts, A. S. \& Gundersen, G. G. mDia mediates Rho-regulated formation and orientation of stable microtubules. Nat. Cell Biol. 3, 723-729 (2001).

26. Frame, M. C. \& Brunton, V. G. Advances in Rho-dependent actin regulation and oncogenic transformation. Curr. Opin. Genet. Dev. 12, 36-43 (2002).

27. Ridley, A. J. Rho family proteins: coordinating cell responses. Trends Cell Biol. 11, 471-477 (2001).

28. Veettil, M. V. et al. RhoA-GTPase facilitates entry of Kaposi's sarcomaassociated herpesvirus into adherent target cells in a Src-dependent manner. J. Virol. 80, 11432-11446 (2006)

29. Weilinger, N. L. et al. Metabotropic NMDA receptor signaling couples Src family kinases to pannexin-1 during excitotoxicity. Nat. Neurosci. 19, 432-442 (2016).

30. Lammers, M., Rose, R., Scrima, A. \& Wittinghofer, A. The regulation of mDial by autoinhibition and its release by Rho*GTP. EMBO J. 24, 4176-4187 (2005).
31. Watanabe, N., Kato, T., Fujita, A., Ishizaki, T. \& Narumiya, S. Cooperation between mDial and ROCK in Rho-induced actin reorganization. Nat. Cell Biol. 1, 136-143 (1999).

32. Alberts, A. S. Identification of a carboxyl-terminal diaphanous-related formin homology protein autoregulatory domain. J. Biol. Chem. 276, 2824-2830 (2001).

33. Wallar, B. J. et al. The basic region of the diaphanous-autoregulatory domain $(\mathrm{DAD})$ is required for autoregulatory interactions with the diaphanous-related formin inhibitory domain. J. Biol. Chem. 281, 4300-4307 (2006).

34. Destaing, O. et al. A novel Rho-mDia2-HDAC6 pathway controls podosome patterning through microtubule acetylation in osteoclasts. J. Cell Sci. 118, 2901-2911 (2005)

35. Li, Y., Shin, D. \& Kwon, S. H. Histone deacetylase 6 plays a role as a distinct regulator of diverse cellular processes. FEBS J. 280, 775-793 (2013).

36. Narita, T., Weinert, B. T. \& Choudhary, C. Functions and mechanisms of non-histone protein acetylation. Nat. Rev. Mol. Cell Biol. 20, 156-174 (2019).

37. Haggarty, S. J., Koeller, K. M., Wong, J. C., Grozinger, C. M. \& Schreiber, S. L. Domain-selective small-molecule inhibitor of histone deacetylase 6 (HDAC6)mediated tubulin deacetylation. Proc. Natl Acad. Sci. USA 100, 4389-4394 (2003).

38. Yoshida, M., Kijima, M., Akita, M. \& Beppu, T. Potent and specific inhibition of mammalian histone deacetylase both in vivo and in vitro by trichostatin A. J. Biol. Chem. 265, 17174-17179 (1990).

39. Amanchy, R., Kalume, D. E. \& Pandey, A. Stable isotope labeling with amino acids in cell culture (SILAC) for studying dynamics of protein abundance and posttranslational modifications. Sci. STKE 2005, pl2 (2005).

40. Heng, T. S. \& Painter, M. W. Immunological Genome Project C. The Immunological Genome Project: networks of gene expression in immune cells. Nat. Immunol. 9, 1091-1094 (2008).

41. Pinheiro, A. R. et al. Histamine induces ATP release from human subcutaneous fibroblasts, via pannexin- 1 hemichannels, leading to $\mathrm{Ca} 2+$ mobilization and cell proliferation. J. Biol. Chem. 288, 27571-27583 (2013).

42. Hubbert, C. et al. HDAC6 is a microtubule-associated deacetylase. Nature 417, 455-458 (2002).

43. Butler, P. L., Staruschenko, A. \& Snyder, P. M. Acetylation stimulates the epithelial sodium channel by reducing its ubiquitination and degradation. $J$. Biol. Chem. 290, 12497-12503 (2015).

44. Alaei, S. R., Abrams, C. K., Bulinski, J. C., Hertzberg, E. L. \& Freidin, M. M. Acetylation of C-terminal lysines modulates protein turnover and stability of Connexin-32. BMC Cell Biol. 19, 22 (2018)

45. Vikram, A. et al. Sirtuin 1 regulates cardiac electrical activity by deacetylating the cardiac sodium channel. Nat. Med. 23, 361-367 (2017).

46. Rajan, S., Plant, L. D., Rabin, M. L., Butler, M. H. \& Goldstein, S. A. Sumoylation silences the plasma membrane leak K+ channel K2P1. Cell 121, 37-47 (2005)

47. Chikumi, H., Vazquez-Prado, J., Servitja, J. M., Miyazaki, H. \& Gutkind, J. S. Potent activation of RhoA by Galpha $\mathrm{q}$ and Gq-coupled receptors. J. Biol. Chem. 277, 27130-27134 (2002).

48. Whyte-Fagundes, P. \& Zoidl, G. Mechanisms of pannexin1 channel gating and regulation. Biochim. Biophys. Acta Biomembr. 1860, 65-71 (2018).

49. Desai, B. N. et al. Cleavage of TRPM7 releases the kinase domain from the ion channel and regulates its participation in Fas-induced apoptosis. Dev. Cell 22, 1149-1162 (2012)

50. Weilinger, N. L., Tang, P. L. \& Thompson, R. J. Anoxia-induced NMDA receptor activation opens pannexin channels via Src family kinases. $J$. Neurosci. 32, 12579-12588 (2012).

51. Lohman, A. W. et al. Pannexin 1 channels regulate leukocyte emigration through the venous endothelium during acute inflammation. Nat. Commun. 6, 7965 (2015).

52. Wang, H. et al. Nicotinic acetylcholine receptor alpha7 subunit is an essential regulator of inflammation. Nature 421, 384-388 (2003).

53. Chavan, S. S., Pavlov, V. A. \& Tracey, K. J. Mechanisms and therapeutic relevance of neuro-immune communication. Immunity 46, 927-942 (2017).

54. Rosas-Ballina, M. et al. Acetylcholine-synthesizing $\mathrm{T}$ cells relay neural signals in a vagus nerve circuit. Science 334, 98-101 (2011).

55. Straub, R. H., Rauch, L., Fassold, A., Lowin, T. \& Pongratz, G. Neuronally released sympathetic neurotransmitters stimulate splenic interferon-gamma secretion from T cells in early type II collagen-induced arthritis. Arthritis Rheum. 58, 3450-3460 (2008).

56. Woehrle, T. et al. Pannexin-1 hemichannel-mediated ATP release together with $\mathrm{P} 2 \mathrm{X} 1$ and $\mathrm{P} 2 \mathrm{X} 4$ receptors regulate $\mathrm{T}$-cell activation at the immune synapse. Blood 116, 3475-3484 (2010).

57. Serrador, J. M. et al. HDAC6 deacetylase activity links the tubulin cytoskeleton with immune synapse organization. Immunity 20, 417-428 (2004).

58. Oh, B. R. et al. Therapeutic effect of a novel histone deacetylase 6 inhibitor, CKD-L, on collagen-induced arthritis in vivo and regulatory $\mathrm{T}$ cells in rheumatoid arthritis in vitro. Arthritis Res. Ther. 19, 154 (2017). 
59. Tsuji, G., Okiyama, N., Villarroel, V. A. \& Katz, S. I. Histone deacetylase 6 inhibition impairs effector CD8 T-cell functions during skin inflammation. $J$. Allergy Clin. Immunol. 135, 1228-1239 (2015).

60. Velasquez, S., Malik, S., Lutz, S. E., Scemes, E. \& Eugenin, E. A. Pannexin1 channels are required for chemokine-mediated migration of CD4+ T lymphocytes: role in inflammation and experimental autoimmune encephalomyelitis. J. Immunol. 196, 4338-4347 (2016).

61. de Zoeten, E. F. et al. Histone deacetylase 6 and heat shock protein 90 control the functions of Foxp3(+) T-regulatory cells. Mol. Cell Biol. 31, 2066-2078 (2011).

62. Good, M. E. et al. Pannexin 1 channels as an unexpected new target of the anti-hypertensive drug spironolactone. Circ. Res. 122, 606-615 (2018).

63. Carrillo, J. J., Pediani, J. \& Milligan, G. Dimers of class A G protein-coupled receptors function via agonist-mediated trans-activation of associated $\mathrm{G}$ proteins. J. Biol. Chem. 278, 42578-42587 (2003).

64. Berg, A. P., Sen, N. \& Bayliss, D. A. TrpC. 3/C. 7 and Slo2.1 are molecular targets for metabotropic glutamate receptor signaling in rat striatal cholinergic interneurons. J. Neurosci. 27, 8845-8856 (2007).

65. Kawaguchi, Y. et al. The deacetylase HDAC6 regulates aggresome formation and cell viability in response to misfolded protein stress. Cell 115, 727-738 (2003).

66. Gao, Y. S., Hubbert, C. C. \& Yao, T. P. The microtubule-associated histone deacetylase 6 (HDAC6) regulates epidermal growth factor receptor (EGFR) endocytic trafficking and degradation. J. Biol. Chem. 285, 11219-11226 (2010).

67. Wang, T., Wei, J. J., Sabatini, D. M. \& Lander, E. S. Genetic screens in human cells using the CRISPR-Cas9 system. Science 343, 80-84 (2014).

68. Kurata, J. S. \& Lin, R. J. MicroRNA-focused CRISPR-Cas9 library screen reveals fitness-associated miRNAs. RNA 24, 966-981 (2018).

69. Yu, R. \& Hinkle, P. M. Signal transduction and hormone-dependent internalization of the thyrotropin-releasing hormone receptor in cells lacking Gq and G11. J. Biol. Chem. 274, 15745-15750 (1999).

70. Devost, D. et al. Conformational profiling of the AT1 angiotensin II receptor reflects biased agonism, G protein coupling, and cellular context. J. Biol. Chem. 292, 5443-5456 (2017).

71. Perez-Riverol, Y. et al. The PRIDE database and related tools and resources in 2019: improving support for quantification data. Nucleic Acids Res. 47, D442-D450 (2019)

\section{Acknowledgements}

This work was supported by P01 HL120840 (to U.M.L., B.N.D., K.S.R., and D.A.B.); by a Pharmacological Sciences Training Grant T32 GM007055 (to E.C.G., J.K.S., C.B.M., and C.A.D.); by the Ministry of Science and Technology, Taiwan (MOST 108-2320-B-007 -007 -MY2, to Y.H.C.); by the National Tsing Hua University, Taiwan, through the Ministry of Education's Higher Education Sprout Project (110Q2705E1 to Y.H.C.); and by predoctoral fellowships from the American Heart Association (PRE223005, to J.K.S.) and the National Cancer Institute (CA236370, to A.K.N.). We thank members of the University of Virginia Pannexin Interest Group for helpful comments throughout the course of the study. We thank Dr. Patricia Hinkle (University of Rochester, Rochester,
NY) and Dr. Asuka Inoue (Tohoku University, Miyagi, Japan) for generously providing cell lines.

\section{Author contributions}

Y.-H.C. and D.A.B. developed concept and designed experiments. Y.-H.C., J.K.S., and A. K.N. performed electrophysiological experiments. Y.-H.C., E.C.G., H-Y.G., and S.Y.G. carried out cell-surface biotinylation and western blotting. C.A.D. performed $\mathrm{Ca}^{2+}$ assay, C.B.M. isolated mouse T lymphocyte and generated PANX1-deleted Jurkat cells using CRISPR-Cas9 technique. M.K. generated Jurkat cell line stably expressing Cas9 proteins Y.-H.C. and H-Y.G. purified PANX1 proteins, and M.Z. and T.P.C. carried out mass spectrometry experiments and the associated data analysis. Y.-H.C., T.P.C., U.M.L., B.N D., K.S.R., and D.A.B. supervised the experiments. Y.-H.C. and D.A.B conceptualized and wrote the manuscript, and all authors edited the manuscript.

\section{Competing interests}

The authors declare no competing interests.

\section{Additional information}

Supplementary information The online version contains supplementary material available at https://doi.org/10.1038/s41467-021-24825-y.

Correspondence and requests for materials should be addressed to Y.-H.C. or D.A.B.

Peer review information Nature Communications thanks Roger Thompson and oter, anonymous, reviewers for their contributions to the peer review of this work. Peer review reports are available.

Reprints and permission information is available at http://www.nature.com/reprints

Publisher's note Springer Nature remains neutral with regard to jurisdictional claims in published maps and institutional affiliations.

\begin{abstract}
(c) (i) Open Access This article is licensed under a Creative Commons Attribution 4.0 International License, which permits use, sharing, adaptation, distribution and reproduction in any medium or format, as long as you give appropriate credit to the original author(s) and the source, provide a link to the Creative Commons license, and indicate if changes were made. The images or other third party material in this article are included in the article's Creative Commons license, unless indicated otherwise in a credit line to the material. If material is not included in the article's Creative Commons license and your intended use is not permitted by statutory regulation or exceeds the permitted use, you will need to obtain permission directly from the copyright holder. To view a copy of this license, visit http://creativecommons.org/ licenses/by/4.0/.
\end{abstract}

(C) The Author(s) 2021 